\title{
RATIONAL BASES FOR SPACES OF HOLOMORPHIC FUNCTIONS IN THE DISC
}

\author{
GEORGE KYRIAZIS AND PENCHO PETRUSHEV
}

\begin{abstract}
A new method for construction of bases for general distribution spaces is developed. This method allows the freedom to prescribe the nature and properties of the basic elements. The method is deployed to the construction of bases consisting of rational functions of uniformly bounded degrees for Besov and Triebel-Lizorkin spaces of holomorphic functions in the unit disc. In turn, this is utilized to give a new proof of Pekarski's direct estimate for rational approximation of holomorphic functions in $H^{p}$.
\end{abstract}

\section{INTRODUCTION}

The main purpose of this paper is to construct bases for spaces of holomorphic functions in the unit disc $D$ in $\mathbb{C}$ consisting of rational functions of uniformly bounded degrees. Such a basis will be of the form

$$
\mathcal{R}=\left\{R_{Q}: Q \in \mathcal{Q}\right\},
$$

where the index set $\mathcal{Q}$ consists of dyadic subintervals of $[0,1]$, quite like Meyer's two hump basis for $H^{p}(D)$ constructed out of periodic wavelets [11]. Each element $R_{Q}$ of $\mathcal{R}$ will be a rational function of degree $\leq K$ that is fixed. Targeted spaces are the Hardy spaces $H^{p}(D)$ and the more general Besov spaces $B_{p, q}^{s}$ and Triebel-Lizorkin spaces $F_{p, q}^{s}$ of holomorphic functions in $D$.

The primary motivation for this undertaking lies in the theory of rational approximation of holomorphic functions in $D$. A. Pekarskii $[15,16]$ proved remarkable direct and inverse estimates for rational approximation in $H^{p}(D)$ which involve Besov spaces and allowed him to completely characterize the rates of rational approximation in $H^{p}(D)$. We refer the reader to [14] for in depth discussion and analysis of these results. The construction of a rational basis as described above will enable us to give another proof of Pekarskii's direct estimate and shed some additional light on rational approximation of holomorphic functions.

To achieve our goal of constructing rational bases for spaces of holomorphic functions we first develop a general method for construction of bases by "small perturbation" of "nice" existing bases. The idea is to approximate the elements of an existing basis, say, $\left\{G_{Q}\right\}$ by the elements of the new basis $\left\{R_{Q}\right\}$ following two simple principles: localization and approximation. The localization of the new basis is measured in terms of the size of the inner products $\left\langle G_{Q}, R_{P}\right\rangle$, while the approximation - by the size of the inner products $\left\langle G_{Q}-R_{Q}, G_{P}\right\rangle$. Technically, it boils down to constructing $\left\{R_{Q}\right\}$ so that the operator with matrix $\left(\left\langle G_{Q}-R_{Q}, G_{P}\right\rangle\right)_{Q, P \in \mathcal{Q}}$ has sufficiently small norms on $\ell^{2}(\mathcal{Q})$ and on the sequence "smoothness" space of interest (e.g. Besov or Triebel-Lizorkin sequence space). This method is rooted in our previous work on bases $[6,7,17]$, where bases were constructed for Besov and Triebel-Lizorkin spaces on $\mathbb{R}^{n}$. In [8] this method was developed for construction 
of frames in a general setting and applied to the construction of frames of small shrinking support on the sphere.

In our specific implementation of the method here we approximate Meyer's two hump wavelet basis functions, denoted $\left\{G_{Q}\right\}$, by appropriate rational functions $\left\{R_{Q}\right\}$ of uniformly bounded degrees on $T=\partial D$. The key observation is that the $H^{2}$ projection of the 1-periodized version of a rational function of the form $\frac{1}{\left(1+x^{2}\right)^{n}}$ on $\mathbb{R}$ or its shifts extends to a rational function on $D$ with poles outside $D$. This allows us to mediate between rational functions with poles outside $D$ and rational functions on $\mathbb{R}$ and achieve the needed localization and approximation properties of the new system.

An important background component of our development is the identification of the Besov and Triebel-Lizorkin spaces $B_{p, q}^{s}$ and $F_{p, q}^{s}$ of holomorphic functions in $D$ as spaces of distributions on the unit circle $T$, obtained by P. Oswald in [12]. We further improve on this result by obtaining a characterization of these Besov and Triebel-Lizorkin spaces in terms of Meyer's two hump wavelet basis [11], quite in the spirit of the wavelet characterization of Besov and Triebel-Lizorkin spaces on $\mathbb{R}$ (see e.g. $[5,11]$ ).

Our main result asserts that under some conditions on the parameters of our rational basis $\mathcal{R}$, it can be used for decomposition of the Besov and Triebel-Lizorkin spaces $B_{p, q}^{s}$ and $F_{p, q}^{s}$ of holomorphic functions in $D$. As an application of this result we prove a direct estimate for $n$-term approximation from our rational basis for $H^{p}$ which involves certain Besov spaces and as a consequence we obtain another proof of Pekarskii's direct theorem [16] for rational approximation in $H^{p}$.

The paper is organized as follows: In $\S 2$ we review Besov and Triebel-Lizorkin spaces of holomorphic functions in $D$ and their identification as spaces of distributions on $T$ from [12]. In $\S 3$ we give the definition of Meyer's two hump wavelet basis for $H^{p}$ and use it for decomposition of Besov and Triebel-Lizorkin spaces on $T$. In $\S 4$ we develop our general method for construction of bases. In $\S 5$ we construct our rational basis and show that it can be used for decomposition of Besov and Triebel-Lizorkin spaces. In $\S 6$ we prove a direct estimate for $n$-term approximation from our rational basis in $H^{p}$ and as a consequence we prove Pekarskii's direct estimate from [16].

Notation: We shall use the notation $T:=\{z \in \mathbb{C}:|z|=1\}$ and $\mathbb{T}:=\mathbb{R} / \mathbb{Z}$; $|x-y|=\min _{n \in \mathbb{Z}}|x-y+n|$ will denote the distance on $\mathbb{T}$ or on $\mathbb{R}$ when dealing with 1-periodic functions. Positive constants will be denoted by $c, c_{1}, c^{\prime}, \ldots$ and they may vary at every occurrence. Also, $a \sim b$ means $c_{1} a \leq b \leq c_{2} a$.

\section{SPACES OF HOLOMORPHIC FUNCTIONS IN THE UNIT DISC}

2.1. Definition of Besov and Triebel-Lizorkin spaces. Here we review Besov and Triebel-Lizorkin spaces of holomorphic functions in the unit disk $D$ in $\mathbb{C}$. We refer the reader to $[13,19,20]$ for the theory of the classical Besov and TriebelLizorkin spaces on $\mathbb{R}^{n}$ and $\mathbb{T}^{n}$. We begin with some notation. We let $A=A(D)$ denote the set of all holomorphic functions in $D$ and for $f \in A(D)$ we shall denote briefly

$$
\|f(r \cdot)\|_{L^{p}}:=\left(\int_{|z|=1}|f(r z)|^{p}|d z|\right)^{1 / p}=\left(2 \pi \int_{0}^{1}\left|f\left(r e^{2 \pi i t}\right)\right|^{p} d t\right)^{1 / p}, \quad 0<p<\infty
$$


and

$$
\|f(r \cdot)\|_{L^{\infty}}:=\sup _{|z|=1}|f(r z)| .
$$

The Hardy space $H^{p}, 0<p \leq \infty$, (see e.g. [22]) is defined as the set of all $f \in A(D)$ such that

$$
\|f\|_{H^{p}}:=\lim _{r \rightarrow 1^{-}}\|f(r \cdot)\|_{L^{p}}<\infty .
$$

For a function $f \in A(D)$ with Taylor series expansion $f(z)=\sum_{n \geq 0} \hat{f}(n) z^{n}$ we set

$$
J^{\beta} f(z):=\sum_{n \geq 0}(n+1)^{\beta} \hat{f}(n) z^{n}, \quad \beta \in \mathbb{R} .
$$

For $\beta>0$ this is called the Weyl derivative of $f$ of order $\beta$.

Definition 2.1. (a) Let $s \in \mathbb{R}$ and $0<p, q \leq \infty$. The Besov space (B-space) $B_{p q}^{s}:=B_{p q}^{s}(A)$ is defined as the set of all functions $f \in A(D)$ with finite semi-norm

$$
\begin{aligned}
\|f\|_{B_{p q}^{s}} & :=\left(\int_{0}^{1}(1-r)^{(\beta-s) q-1}\left\|J^{\beta} f(r \cdot)\right\|_{L^{p}}^{q} d r\right)^{1 / q} \text { if } q \neq \infty, \text { and } \\
\|f\|_{B_{p \infty}^{s}} & :=\sup _{0<r<1}(1-r)^{\beta-s}\left\|J^{\beta} f(r \cdot)\right\|_{L^{p}} \quad \text { if } q=\infty .
\end{aligned}
$$

(b) Let $s \in \mathbb{R}, 0<p<\infty$, and $0<q \leq \infty$. The Triebel-Lizorkin space (F-space) $F_{p q}^{s}=F_{p q}^{s}(A)$ is defined as the set of all $f \in A(D)$ with finite quasinorm

$$
\begin{aligned}
\|f\|_{F_{p q}^{s}} & :=\left\|\left(\int_{0}^{1}(1-r)^{(\beta-s) q-1}\left|J^{\beta} f(r \cdot)\right|^{q} d r\right)^{1 / q}\right\|_{L^{p}} \quad \text { if } q \neq \infty, \text { and } \\
\|f\|_{F_{p \infty}^{s}} & :=\left\|\sup _{0<r<1}(1-r)^{\beta-s}\left|J^{\beta} f(r \cdot)\right|\right\|_{L^{p}} .
\end{aligned}
$$

In the definition of all of the above quasi-norms $\beta:=s+1$.

Observe that in the above definition it suffices to require $\beta>s$. For such a selection of $\beta$ the respective quasinorms are equivalent. For this and other properties of the B- and F-spaces we refer the reader to $[2,12]$.

It will be important for our further developments that the Besov and TriebelLizorkin spaces of holomorphic functions defined above can be identified as spaces of distributions on the unit circle $T=\{z:|z|=1\}$, which should be viewed as their boundary values.

We denote by $\mathcal{D}_{+}$the class of test functions $\phi: T \rightarrow \mathbb{C}$ of the form

$$
\phi\left(e^{2 \pi i x}\right)=\sum_{n \geq 0} \hat{\phi}(n) e^{2 \pi i n x}, \quad x \in[0,1), \quad \text { with } \hat{\phi}(n):=\int_{0}^{1} \phi\left(e^{2 \pi i x}\right) e^{-2 \pi i n x} d x
$$

such that

$$
\mathcal{P}_{r}(\phi):=\sup _{n \geq 0}(n+1)^{r}|\hat{\phi}(n)|<\infty \quad \text { for all } \quad r \geq 0 .
$$

The locally convex topology in $\mathcal{D}_{+}$is defined by the norms $\mathcal{P}_{r}, r=0,1, \ldots$ The space of distributions $\mathcal{D}_{+}^{\prime}$ is defined as the set of all bounded linear functionals on $\mathcal{D}_{+}$. The pairing of $f \in \mathcal{D}_{+}^{\prime}$ and $\phi \in \mathcal{D}_{+}$will be denoted by $\langle f, \phi\rangle:=f(\bar{\phi})$, which is consistent with the inner product $\langle f, g\rangle=\int_{0}^{1} f\left(e^{2 \pi i x}\right) \overline{g\left(e^{2 \pi i x}\right)} d x$ on $T$.

The above definition readily implies that for any $f \in \mathcal{D}_{+}^{\prime}$ there exists $r \geq 0$ such that $|\langle f, \phi\rangle| \leq c_{r} \mathcal{P}_{r}(\phi)$ for all $\phi \in \mathcal{D}_{+}$, which yields

$$
|\hat{f}(n)| \leq c_{r}(n+1)^{r}, \quad n \geq 0, \quad \text { where } \hat{f}(n):=\left\langle f, e^{2 \pi i n x}\right\rangle .
$$


This in turn leads to the conclusion that for any $f \in \mathcal{D}_{+}^{\prime}$

$$
f=\sum_{n \geq 0} \hat{f}(n) e^{2 \pi i n x} \quad \text { in } \quad \mathcal{D}_{+}^{\prime} .
$$

Therefore, for any $f \in \mathcal{D}_{+}^{\prime}$ there is a holomorphic function $f \in A(D)$ (the extension of $f$ ) such that

$$
f(z)=\sum_{n \geq 0} \hat{f}(n) z^{n}, \quad|z|<1 .
$$

Conversely, for any function $f \in A(D)$ with at most polynomially growing Taylor coefficients (like in (2.2)) there is a unique distribution $f \in \mathcal{D}_{+}^{\prime}$ with the same Fourier series coefficients.

New convention. To simplify our notation, from now on we shall use the notation $\phi(x)$ and $f(x)$ instead of $\phi\left(e^{2 \pi i x}\right)$ and $f\left(e^{2 \pi i x}\right)$, and consider the functions defined on $\mathbb{R}$ and 1-periodic, i.e. defined on $\mathbb{T}:=\mathbb{R} / \mathbb{Z}$. For instance, the class of test functions $\mathcal{D}_{+}$will consist of all functions $\phi: \mathbb{T} \rightarrow \mathbb{C}$ of the form

$$
\phi(x)=\sum_{n \geq 0} \hat{\phi}(n) e^{2 \pi i n x} \quad \text { with } \quad \hat{\phi}(n):=\int_{0}^{1} \phi(x) e^{-2 \pi i n x} d x
$$

such that $\mathcal{P}_{r}(\phi)<\infty$ for all $r \geq 0$, where the norms $\mathcal{P}_{r}$ are defined in (2.1). It is easy to see that the topology on $\mathcal{D}_{+}$can be equivalently defined by the semi-norms

$$
\mathcal{N}_{r}(\phi):=\left\|\phi^{(r)}\right\|_{L^{\infty}}, \quad r=0,1, \ldots
$$

By (2.3) it follows that for $f \in \mathcal{D}_{+}^{\prime}$ and $\phi \in \mathcal{D}_{+}$

$$
\langle f, \phi\rangle=\sum_{n \geq 0} \hat{f}(n) \overline{\hat{\phi}(n)}
$$

where the series converges absolutely.

As already inducated in the introduction, when dealing with 1-periodic functions on $\mathbb{R}$ we shall use the distance $|x-y|=\min _{n \in \mathbb{Z}}|x-y+n|$ on $\mathbb{R}$ or $\mathbb{T}$.

As usual for $\phi \in \mathcal{D}_{+}$and $x \in \mathbb{R}$ we denote by $\tau_{x}$ the translation operator $\tau_{x} \phi(\cdot):=\phi(\cdot-x)$ and we set $\tilde{\phi}(\cdot):=\phi(-\cdot)$. These definitions extend by duality to $\mathcal{D}_{+}^{\prime}$. In particular, for $f \in \mathcal{D}_{+}^{\prime}$ and $\phi \in \mathcal{D}_{+}$we define the convolution of $f$ with $\phi$ by $f * \phi(x):=f\left(\tau_{x}(\tilde{\phi})\right)$. It is easily seen that $f * \phi \in \mathcal{D}_{+}$and

$$
f * \phi(x)=\sum_{n \geq 0} \hat{f}(n) \hat{\phi}(n) e^{2 \pi i n x} .
$$

2.2. Equivalent definition of Besov and Triebel-Lizorkin spaces. Here we give an equivalent definition for the B- and F-spaces, introduced by Definition 2.1, in terms of distributions on $\mathbb{T}$. We shall essentially follow the development of these spaces in [12]. Let $\hat{\varphi} \in C^{\infty}[0, \infty)$ satisfy

$$
\operatorname{supp} \hat{\varphi} \subset[0,2], \quad \hat{\varphi} \geq 0, \quad \hat{\varphi}(t)=1 \text { if } t \in[0,1] .
$$

Set $\hat{\varphi}_{1}(t):=\hat{\varphi}(t)-\hat{\varphi}(2 t)$ and note that $\operatorname{supp} \hat{\varphi}_{1} \subset[1 / 2,2]$.

Let the trigonometric polynomials $\Phi_{j}$ be defined by

$$
\Phi_{0}(x):=1 \quad \text { and } \quad \Phi_{j}(x):=\sum_{\nu=0}^{\infty} \hat{\varphi}_{1}\left(2^{-j+1} \nu\right) e^{2 \pi i \nu x}, j \geq 1 .
$$


Definition 2.2. (a) Let $s \in \mathbb{R}$ and $0<p, q \leq \infty$. The Besov space $B_{p q}^{s}=B_{p q}^{s}\left(\mathcal{D}_{+}^{\prime}\right)$ is defined as the set of all $f \in \mathcal{D}_{+}^{\prime}$ such that

$$
\|f\|_{B_{p q}^{s}}:=\left(\sum_{j=0}^{\infty}\left(2^{s j}\left\|\Phi_{j} * f\right\|_{L_{p}}\right)^{q}\right)^{1 / q}<\infty
$$

with the usual modification when $q=\infty$.

(b) Let $s \in \mathbb{R}, 0<p<\infty$, and $0<q \leq \infty$. The Triebel-Lizorkin space $F_{p q}^{s}=F_{p q}^{s}\left(\mathcal{D}_{+}^{\prime}\right)$ is defined as the set of all $f \in \mathcal{D}_{+}^{\prime}$ such that

$$
\|f\|_{F_{p q}^{s}}:=\left\|\left(\sum_{j=0}^{\infty}\left(2^{s j}\left|\Phi_{j} * f(\cdot)\right|\right)^{q}\right)^{1 / q}\right\|_{L^{p}}<\infty
$$

with the usual modification when $q=\infty$.

One of the main results in [12] asserts that Definitions 2.1 and 2.2 essentially define the same spaces:

Theorem 2.3. [12] With the identification from (2.3) - (2.4) the Besov spaces from Definition 2.1 and Definition 2.2 are the same with equivalent norms. The same is true for the Triebel-Lizorkin spaces from Definition 2.1 and Definition 2.2.

Several remarks are in order: 1) The spaces $B_{p q}^{s}$ and $F_{p q}^{s}$ are in general quasiBanach spaces and Banach spaces if $p, q \geq 1$.

2) In the definition of the Besov and Triebel-Lizorkin norms above $\hat{\varphi}_{1}$ can be replaced by any $\hat{\varphi}_{1} \in C^{\infty}[0, \infty)$ with the properties: $\operatorname{supp} \hat{\varphi}_{1} \subset[\delta, K]$ and

$$
\left|\hat{\varphi}_{1}(t)\right| \geq c>0 \text { for } \delta+\varepsilon \leq t \leq K-\varepsilon,
$$

where $0<\delta<1, K>1, \varepsilon>0$, and $2(\delta+\varepsilon) \leq K-\varepsilon$. The resulting norms are equivalent.

3) The spaces $B_{p q}^{s}$ and $F_{p q}^{s}$ are continuously embedded in $\mathcal{D}_{+}^{\prime}$, i.e. for any $f \in B_{p q}^{s}$ there exists $k \geq 0$ such that $|\langle f, \phi\rangle| \leq c\|f\|_{B_{p q}^{s}} \mathcal{P}_{k}(\phi)$ for all $\phi \in \mathcal{D}_{+}$, and the same holds for $F_{p q}^{s}$.

4) The Hardy space $H^{p}$ can be identified as $F_{p 2}^{0}$ with equivalent norms, when $0<p<\infty$.

\subsection{Additional background material.}

The maximal operator $\mathcal{M}_{t}, 0<t<\infty$, is defined by

$$
\mathcal{M}_{t} f(x):=\sup _{I \ni x}\left(\frac{1}{|I|} \int_{I}|f(y)|^{t} d y\right)^{1 / t}
$$

where the sup is over all intervals $I$ of length $|I| \leq 1$.

The Fefferman-Stein (see [18]) vector-valued maximal inequality will play a prominent role: If $0<p<\infty, 0<q \leq \infty$ and $0<t<\min \{p, q\}$ then for any sequence of functions $\left\{f_{\nu}\right\}_{\nu=1}^{\infty}$ on $\mathbb{T}$,

$$
\left\|\left(\sum_{\nu=1}^{\infty}\left|\mathcal{M}_{t} f_{\nu}(\cdot)\right|^{q}\right)^{1 / q}\right\|_{L^{p}} \leq c\left\|\left(\sum_{\nu=1}^{\infty}\left|f_{\nu}(\cdot)\right|^{q}\right)^{1 / q}\right\|_{L^{p}} .
$$

An estimate on $\left(\mathcal{M}_{t} \mathbb{1}_{I}\right)(x)$ will be needed for an arbitrary interval $I \subset \mathbb{R}$ of length $|I|<1$, where $\mathbb{1}_{I}$ is the 1-periodic extension of the characteristic function on $I$. 
Lemma 2.4. For any interval $I=[a, b]$ of length $b-a<1$

$$
\left(\mathcal{M}_{t} \mathbb{1}_{I}\right)(x) \sim\left(1+|I|^{-1}|x-a|\right)^{-1 / t},
$$

where as elsewhere $|x-a|:=\min _{n \in \mathbb{Z}}|x-a+n|$.

This lemma is trivial and the proof will be omitted.

Localization of trigonometric polynomials. The nearly exponential localization of trigonometric polynomials with coefficients obtained from sampling of smooth compactly supported cutoff functions will be needed.

Lemma 2.5. Given $\hat{\varphi} \in C^{\infty}(\mathbb{R})$ with $\operatorname{supp} \hat{\varphi} \subset[c, d]$ and $n \geq 1$ we write

$$
\Phi(x):=\sum_{\nu \in \mathbb{Z}} \hat{\varphi}(\nu / n) e^{2 \pi i \nu x} .
$$

Then for any $r \geq 0$ and $\sigma>0$ there exists a constant $c>0$ such that

$$
\left|\Phi_{n}^{(r)}(x)\right| \leq c n^{r+1}(1+n|x|)^{-\sigma}, \quad|x| \leq 1 / 2 .
$$

This lemma is well known and follows by the fact that $\varphi$, the inverse Fourier transform of the function $\hat{\varphi}$ above, belongs to the Schwartz class $\mathcal{S}(\mathbb{R})$ of rapidly decaying $C^{\infty}$ functions on $\mathbb{R}$. For the proof one just applies the Poisson summation formula as in the proof of Lemma 5.9 below. We omit the details.

\section{Decomposition of B- And F-Spaces Via the two hump Basis}

3.1. Two hump wavelet basis. We next introduce wavelets with two humps following closely Meyer's construction from [11]. As in [11], given $f \in L^{1}(\mathbb{R})$ we denote by $\hat{f}$ its Fourier transform, defined by

$$
\hat{f}(\xi):=\int_{\mathbb{R}} f(x) e^{-i x \xi} d x .
$$

Let $\Psi:=\left\{2^{j / 2} \psi\left(2^{j} x-k\right), j, k \in \mathbb{Z}\right\}$ be Meyer's orthonormal wavelet basis for $L^{2}(\mathbb{R})$. We recall that $\psi$ is a real-valued function with the properties:

$$
\begin{aligned}
& \psi \in \mathcal{S}(\mathbb{R}) \quad \text { with } \quad \text { supp } \hat{\psi} \subset\left\{\xi: \frac{2 \pi}{3} \leq|\xi| \leq \frac{8 \pi}{3}\right\}, \\
& \psi(1-x)=\psi(x), \\
& \sum_{j \in \mathbb{Z}} \mid \hat{\psi}\left(\left.\xi 2^{-j}\right|^{2}=1, \xi \neq 0 .\right.
\end{aligned}
$$

From (3.3) it follows that $\psi(x+1 / 2)$ is even. It is convenient to write

$$
\hat{\psi}(\xi)=\omega(\xi) e^{-i \xi / 2},
$$

where $\omega(\xi)=\psi(\widehat{\cdot+1} / 2)(\xi)$ is also real-valued and even.

Set

$$
g_{j, k}(x):=2^{j / 2} \sum_{\ell \in \mathbb{Z}} \psi\left(2^{j}(x+\ell)-k\right), \quad 0 \leq k<2^{j}, j \geq 0 .
$$

It is not hard to see that the family $\{1\} \cup\left\{g_{j, k}: 0 \leq k<2^{j}, j \geq 0\right\}$ is an orthonormal basis for $L^{2}(\mathbb{T})$. These are the 1-periodic Meyer's wavelets [11]. Then $g_{j, k}(x)+g_{j, k}(-x)$ is even and noting that $g_{j, k}(-x)=g_{j, k}(1-x)=g_{j, k^{*}}(x)$ with $k^{*}=2^{j}-k-1$ we conclude that the family

$$
G:=\{1\} \cup\left\{g_{j, k}+g_{j, k^{*}}: 0 \leq k<2^{j-1}, j \geq 0\right\}
$$


is an orthogonal basis for the subspace of even functions in $L^{2}(\mathbb{T})$.

Following Bochkariev's idea from [1] Meyer constructed a basis for $H^{2}$ by projecting the elements of $G$ onto $H^{2}$. More precisely, using the Poisson summation formula it readily follows that

$$
g_{j, k}(x)=2^{-j / 2} \sum_{\nu \in \mathbb{Z}} \hat{\psi}\left(2 \pi \nu 2^{-j}\right) e^{2 \pi i \nu\left(x-k 2^{-j}\right)} .
$$

Set

$$
\begin{aligned}
G_{j, k}(x) & :=2^{-j / 2} \sum_{\nu \geq 0} \hat{\psi}\left(2 \pi \nu 2^{-j}\right)\left(e^{2 \pi i \nu\left(x-k 2^{-j}\right)}+e^{2 \pi i \nu\left(x-k^{*} 2^{-j}\right)}\right) \\
& =2^{-j / 2} \sum_{\nu \geq 0} \omega\left(2 \nu \pi 2^{-j}\right) \cos \left(\frac{2 \nu \pi}{2^{j}}\left(k+\frac{1}{2}\right)\right) e^{2 \pi i \nu x}
\end{aligned}
$$

for $0 \leq k<2^{j-1}, j \geq 0$. This is the orthogonal projection of $g_{j, k}+g_{j, k^{*}}$ onto $H^{2}(\mathbb{T})$. Note that $G_{0,0}(x)=-e^{2 \pi i x}$. In addition, let $G_{-1,0}(x):=1$. Then the family

$$
\left\{G_{j, k}: 0 \leq k<2^{j-1}, j \geq-1\right\}
$$

is an orthonormal basis for $H^{2}$. Furthermore, as is shown in [11] this is an unconditional basis for $H^{p}$ for $0<p<\infty$ and a Schauder basis for $H^{\infty}$.

We next introduce more compact and convenient for us notation. Let

$$
Q_{j k}:=\left[k / 2^{j},(k+1) / 2^{j}\right), \quad k=0, \ldots, 2^{j-1}-1, j \geq 1,
$$

and if $Q=Q_{j, k}$ we let $x_{Q}:=k / 2^{j}$ denote the left end of $Q$ and $\ell(Q):=2^{-j}$ is its length. Also, if $Q=Q_{j k}$ we set $Q^{*}=Q_{j k^{*}}$, where $k^{*}:=2^{j}-k-1$.

We define for $j \geq 1$

$$
\mathcal{Q}_{j}:=\left\{Q_{j k}: 0 \leq k<2^{j-1}\right\}, \quad \mathcal{Q}_{j}^{*}:=\left\{Q_{j k^{*}}: 0 \leq k<2^{j-1}\right\}=\left\{Q^{*}: Q \in \mathcal{Q}_{j}\right\},
$$

$\mathcal{V}_{j}:=\mathcal{Q}_{j} \cup \mathcal{Q}_{j}^{*}$, and we also set $\mathcal{Q}_{0}=\mathcal{Q}_{-1}:=\{[0,1]\}, \mathcal{Q}_{0}^{*}=\mathcal{Q}_{-1}^{*}:=\emptyset$. Note that $\mathcal{Q}_{j} \cap \mathcal{Q}_{j}^{*}=\emptyset$. Finally we define

$$
\mathcal{Q}:=\cup_{j \geq-1} \mathcal{Q}_{j} \quad \text { and } \quad \mathcal{V}:=\cup_{j \geq-1} \mathcal{V}_{j}
$$

In what follows we shall identify any pair of indices $(j, k)$ with the respective dyadic interval $Q_{j k}=\left[k / 2^{j}, k+1 / 2^{j}\right)$. Then we can write

$$
\mathcal{G}=\left\{G_{Q}: Q \in \mathcal{Q}\right\} .
$$

Observe that by Lemma 2.5 it readily follows that the basis elements $G_{Q}$ have a two hump nearly exponential localization: For any $\sigma>0$ there exists a constant $c_{\sigma}>0$ such that for any $Q \in \mathcal{Q}$

$$
\left|G_{Q}(x)\right| \leq c_{\sigma} \ell(Q)^{-1 / 2}\left(\left(1+2^{j}\left|x-x_{Q}\right|\right)^{-\sigma}+\left(1+2^{j}\left|x-x_{Q^{*}}\right|\right)^{-\sigma}\right) .
$$

It is also not hard to estimate the norms of $G_{Q}$. Indeed, $\left\|G_{Q}\right\|_{L^{2}}=1$ by construction. This and (3.9) lead to

$$
\left\|G_{Q}\right\|_{L^{p}} \sim \ell(Q)^{-1 / 2+1 / p} \text { for } \quad 0<p \leq \infty,
$$

see e.g. the proof of Proposition 5.12 below.

We next show that that $\mathcal{G}$ is a decomposition system for $\mathcal{D}_{+}$and $\mathcal{D}_{+}^{\prime}$. 
Proposition 3.1. For any $\phi \in \mathcal{D}_{+}$

$$
\phi=\sum_{Q \in \mathcal{Q}}\left\langle\phi, G_{Q}\right\rangle G_{Q} \quad \text { in } \quad \mathcal{D}_{+}
$$

and, hence, for any $f \in \mathcal{D}_{+}^{\prime}$

$$
f=\sum_{Q \in \mathcal{Q}}\left\langle f, G_{Q}\right\rangle G_{Q} \quad \text { in } \quad \mathcal{D}_{+}^{\prime} .
$$

Proof. Since $\mathcal{G}$ is a basis for $H^{2}$ to prove (3.11) it suffices to show that the series in (3.11) converges in the topology of $\mathcal{D}_{+}$.

Let $r \geq 0$ and choose $\ell>r+1$. Using (3.5) and (2.1) if $Q=Q_{j k}, 0 \leq k<2^{j-1}$, $j \geq 0$, we obtain

$$
\begin{aligned}
\left|\left\langle\phi, G_{Q}\right\rangle\right| & \leq 2^{-j / 2} \sum_{2^{j} / 3<\nu<2^{j+2} / 3}\left|\hat{\psi}\left(2 \pi \nu 2^{-j}\right)\right||\hat{\phi}(\nu)| \\
& \leq 2^{-j / 2}\|\hat{\psi}\|_{\infty} \mathcal{P}_{\ell}(\phi) \sum_{2^{j} / 3<\nu<2^{j+2} / 3}(\nu+1)^{-\ell} \leq c 2^{-j(\ell-1 / 2)} \mathcal{P}_{\ell}(\phi)
\end{aligned}
$$

and evidently $\left|\hat{G}_{Q}(n)\right| \leq 2^{-j / 2+1}\left|\hat{\psi}\left(2 \pi n 2^{-j}\right)\right|$. Hence

$$
\sum_{Q \in \mathcal{Q}_{j}}\left|\left\langle\phi, G_{Q}\right\rangle\right|\left|\hat{G}_{Q}(n)\right| \leq c 2^{-j(\ell-1)}\left|\hat{\psi}\left(2 \pi n 2^{-j}\right)\right| \mathcal{P}_{\ell}(\phi) .
$$

From this and (3.2) we infer

$$
(n+1)^{r} \sum_{Q \in \mathcal{Q}_{j}}\left|\left\langle\phi, G_{Q}\right\rangle\right|\left|\hat{G}_{Q}(n)\right| \leq c 2^{-j(\ell-r-1)}\left|\hat{\psi}\left(2 \pi n 2^{-j}\right)\right| \mathcal{P}_{\ell}(\phi), \quad n \geq 0 .
$$

Further, we use again (3.2) to obtain

$$
\begin{aligned}
\sup _{n \geq 0}(n+1)^{r} \sum_{j \geq N} \sum_{Q \in \mathcal{Q}_{j}}\left|\left\langle\phi, G_{Q}\right\rangle\right|\left|\hat{G}_{Q}(n)\right| & \leq c \mathcal{P}_{\ell}(\phi) \sup _{n \geq 0} \sum_{j \geq N} 2^{-j(\ell-r-1)}\left|\hat{\psi}\left(2 \pi n 2^{-j}\right)\right| \\
& \leq c \mathcal{P}_{\ell}(\phi) 2^{-N(\ell-r-1)} \rightarrow 0 \text { as } N \rightarrow \infty
\end{aligned}
$$

which readily implies the convergence in $\mathcal{D}_{+}$of the series in (3.11).

3.2. Main assertion. In order to show that $\mathcal{G}$ is a basis for Besov and TribelLizorkin spaces we need to introduce their sequence counterparts.

Definition 3.2. (a) Given $s \in \mathbb{R}$ and $0<p, q \leq \infty$ the space $b_{p q}^{s}:=b_{p q}^{s}(\mathcal{Q})$ is defined as the set of all complex-valued sequences $h:=\left\{h_{Q}\right\}_{Q \in \mathcal{Q}}$ such that

$$
\|h\|_{b_{p q}^{s}}:=\left(\sum_{j=-1}^{\infty} 2^{j\left(s-\frac{1}{p}+\frac{1}{2}\right) q}\left(\sum_{Q \in \mathcal{Q}_{j}}\left|h_{Q}\right|^{p}\right)^{\frac{q}{p}}\right)^{1 / q}<\infty
$$

with the usual modification for $q=\infty$.

(b) Given $s \in \mathbb{R}, 0<p<\infty$, and $0<q \leq \infty$ the space $f_{p q}^{s}:=f_{p q}^{s}(\mathcal{Q})$ is defined as the space of all complex-valued sequences $h:=\left\{h_{Q}\right\}_{Q \in \mathcal{Q}}$ such that

$$
\|h\|_{f_{p q}^{s}}:=\left\|\left(\sum_{j=-1}^{\infty} 2^{s j q} \sum_{Q \in \mathcal{Q}_{j}}\left[\left|h_{Q}\right| \tilde{\mathbb{1}}_{Q}(\cdot)\right]^{q}\right)^{1 / q}\right\|_{L^{p}}<\infty
$$

with the usual modification for $q=\infty$. Here $\tilde{\mathbb{1}}_{Q}:=\ell(Q)^{-1 / 2} \mathbb{1}_{Q}$. 
We next use the above sequence spaces to establish the claimed decomposition result.

Theorem 3.3. (a) Let $s \in \mathbb{R}, 0<p, q \leq \infty$. Then any $f \in B_{p q}^{s}$ has a unique representation

$$
f=\sum_{Q \in \mathcal{Q}} c_{Q}(f) G_{Q}, \quad \text { where } c_{Q}(f):=\left\langle f, G_{Q}\right\rangle
$$

and the convergence is in the norm of $B_{p q}^{s}$; the convergence is unconditional if $p, q \neq \infty$. Moreover,

$$
\|f\|_{B_{p q}^{s}} \sim\left\|\left(c_{Q}(f)\right)_{Q}\right\|_{b_{p q}^{s}(\mathcal{Q})}
$$

(b) Let $s \in \mathbb{R}, 0<p<\infty, 0<q \leq \infty$. Then any $f \in F_{p q}^{s}$ has a unique representation

$$
f=\sum_{Q \in \mathcal{Q}} c_{Q}(f) G_{Q}, \quad \text { where } c_{Q}(f):=\left\langle f, G_{Q}\right\rangle
$$

and the convergence is in $F_{p q}^{s}$; the convergence is unconditional if $q \neq \infty$. Furthermore,

$$
\|f\|_{F_{p q}^{s}} \sim\left\|\left(c_{Q}(f)\right)_{Q}\right\|_{f_{p q}^{s}(\mathcal{Q})}
$$

This theorem is quite close in spirit to the developments in $[3,4,5,11,19]$. For completeness we provide the essential parts of its proof in the following. We shall need several lemmas.

Lemma 3.4. For any $\sigma>0$ there exists a constant $c_{\sigma}>0$ such that

$$
\left|\Phi_{j} * G_{Q}(x)\right| \leq c_{\sigma} 2^{\nu / 2}\left(\left(1+2^{\nu}\left|x-x_{Q}\right|\right)^{-\sigma}+\left(1+2^{\nu}\left|x-x_{Q^{*}}\right|\right)^{-\sigma}\right)
$$

for $Q \in \mathcal{Q}_{\nu}, j-2 \leq \nu \leq j+1$, and $\Phi_{j} * G_{Q}(x)=0$ for $Q \in \mathcal{Q}_{\nu}$ whenever $\nu \geq j+2$ or $\nu \leq j-3$. Here $\mathcal{Q}_{\nu}:=\emptyset$ if $\nu<-1$.

Proof. Let $Q=Q_{\nu k}, j-2 \leq \nu \leq j+1, j \geq 1$, and $\nu \geq 0$. By the definitions of $\Phi_{j}, G_{Q}$ in (2.7),(3.5), and by (2.5) we get

$$
\begin{aligned}
\Phi_{j} * G_{Q}(x) & =2^{-\nu / 2} \sum_{\mu \geq 0} \hat{\varphi}_{1}\left(\frac{\mu}{2^{j-1}}\right) \hat{\psi}\left(\frac{2 \pi \mu}{2^{\nu}}\right) e^{2 \pi i \mu\left(x-x_{Q}\right)} \\
& +2^{-\nu / 2} \sum_{\mu \geq 0} \hat{\varphi}_{1}\left(\frac{\mu}{2^{j-1}}\right) \hat{\psi}\left(\frac{2 \pi \mu}{2^{\nu}}\right) e^{2 \pi i \mu\left(x-x_{Q^{*}}\right)}=: F(x)+F^{*}(x) .
\end{aligned}
$$

Set $\hat{g}(\xi):=\hat{\varphi}_{1}\left(2^{\nu-j+1} \xi\right) \hat{\psi}(2 \pi \xi)$. Evidently, $\hat{g} \in C^{\infty}(\mathbb{R})$, supp $\hat{g} \subset[1 / 3,4 / 3]$, and all derivatives of $\hat{g}$ can be bounded by constants independent of $j, \nu$ due to $|\nu-j| \leq 3$. On the other hand, $\hat{\varphi}_{1}\left(\frac{\mu}{2^{j-1}}\right) \hat{\psi}\left(\frac{2 \pi \mu}{2^{\nu}}\right)=\hat{g}\left(\frac{\mu}{2^{\nu}}\right)$. Therefore, by Lemma 2.5 we get

$$
|F(x)| \leq c_{\sigma} 2^{\nu / 2}\left(1+2^{\nu}\left|x-x_{Q}\right|\right)^{-\sigma}
$$

and the same estimate with $x_{Q}$ replaced by $x_{Q^{*}}$ holds for $F^{*}$. These two estimates yield (3.19). In the case when $j=0$ or $\nu=-1$, estimate (3.19) holds trivially.

Also, $\Phi_{j} * G_{Q}(x)=0$ for $Q \in \mathcal{Q}_{\nu}, \nu \geq j+2$ or $\nu \leq j-3$, since in this case $\hat{g} \equiv 0$ with $\hat{g}$ from above. 
Definition 3.5. For a collection of complex numbers $\left\{h_{Q}\right\}_{Q \in \mathcal{V}_{j}}$ we set

$$
h_{Q}^{\natural}:=\sum_{P \in \mathcal{V}_{j}} \frac{\left|h_{P}\right|}{\left.\left(1+2^{j}\left|x_{P}-x_{Q}\right|\right)\right)^{\kappa}} .
$$

Here $\kappa>0$ is a sufficiently large parameter that will be selected later on.

Lemma 3.6. Suppose $t>0$ and let $\left\{h_{Q}\right\}_{Q \in \mathcal{V}_{j}}, j \geq 0$, be a collection of complex numbers. Assume $\kappa>1 / t+1$ in the definition (3.20) of $h_{Q}^{\natural}$. Then

$$
h_{Q}^{\natural} \mathbb{1}_{Q}(x) \leq c \mathcal{M}_{t}\left(\sum_{P \in \mathcal{V}_{j}}\left|h_{P}\right| \mathbb{1}_{P}\right)(x) .
$$

This lemma is essentially the univariate version of Lemma A.2 in [4] and its proof will be omitted.

Lemma 3.7. For any $t>0$ there exists a constant $c>0$ such that for any trigonometric polynomial $g$ of degree $\leq n(n \geq 0)$ one has

$$
\sup _{y \in \mathbb{T}} \frac{|g(y)|}{(1+n|x-y|)^{1 / t}} \leq c\left(\mathcal{M}_{t} g\right)(x), \quad x \in \mathbb{T} .
$$

This lemma is well known; its version for entire functions of exponential type is given in [19], Theorem 1.3.1. We omit the proof.

Proof of Theorem 3.3. We shall only prove part (b) of this theorem in the case when $q<\infty$; the proof in the case $q=\infty$ and the proof of part (a) are easier and will be omitted.

Choose the constants $t, \kappa, \sigma$ so that $0<t<\min \{p, q\}, \kappa>1 / t+1$, and $\sigma \geq \kappa$. Let $f \in F_{p q}^{s}$. Then by Proposition $3.1 f=\sum_{Q \in \mathcal{Q}}\left\langle f, G_{Q}\right\rangle G_{Q}$ in $\mathcal{D}_{+}^{\prime}$. Denote briefly $c_{Q}:=\left\langle f, G_{Q}\right\rangle$ and let us extend the sequence $\left(c_{Q}\right)_{Q \in \mathcal{Q}}$ to $\mathcal{Q}^{*}$ by setting $c_{Q^{*}}:=c_{Q}$.

From above and (3.19) we get

$$
\begin{aligned}
\left|\Phi_{j} * f(x)\right| & =\left|\sum_{Q \in \mathcal{Q}} c_{Q} \Phi_{j} * G_{Q}(x)\right| \leq \sum_{j-2 \leq \nu \leq j+1} \sum_{Q \in \mathcal{Q}_{\nu}}\left|c_{Q}\right|\left|\Phi_{j} * G_{Q}(x)\right| \\
& \leq c \sum_{j-2 \leq \nu \leq j+1} 2^{\nu / 2} \sum_{Q \in \mathcal{Q}_{\nu}} \frac{\left|c_{Q}\right|}{\left(1+2^{\nu}\left|x-x_{Q}\right|\right)^{\sigma}}+\frac{\left|c_{Q}\right|}{\left(1+2^{\nu}\left|x-x_{Q^{*}}\right|\right)^{\sigma}} \\
& =c \sum_{j-2 \leq \nu \leq j+1} 2^{\nu / 2} \sum_{Q \in \mathcal{V}_{\nu}} \frac{\left|c_{Q}\right|}{\left(1+2^{\nu}\left|x-x_{Q}\right|\right)^{\sigma}} .
\end{aligned}
$$

For any $Q \in \mathcal{V}_{j}$ we denote $\mathcal{W}_{Q}:=\left\{P \in \cup_{j-2 \leq \nu \leq j+1} \mathcal{V}_{\nu}: P \cap Q \neq \emptyset\right\}$, where $\mathcal{V}_{\nu}:=\emptyset$ if $\nu<0$. Note that $\# \mathcal{W}_{Q} \leq 5$. Then it follows from above that

$$
\left|\Phi_{j} * f(x)\right| \leq c \sum_{P \in \mathcal{W}_{Q}} c_{P}^{\natural} \tilde{\mathbb{1}}_{P}(x), \quad x \in Q \in \mathcal{V}_{j}
$$

Here $\tilde{\mathbb{1}}_{P}:=\ell(P)^{-1 / 2} \mathbb{1}_{P}$ is the $L^{2}$-normalized characteristic function of $P$. We now insert the above in (2.9) and use Lemma 3.6 and the maximal inequality (2.11) to 
obtain

$$
\begin{aligned}
\|f\|_{F_{p q}^{s}} & \leq c\left\|\left(\sum_{j \geq 0}\left[2^{s j} \sum_{Q \in \mathcal{V}_{j}} \sum_{P \in \mathcal{W}_{Q}} c_{P}^{\natural} \tilde{\mathbb{1}}_{P}(\cdot)\right]^{q}\right)^{1 / q}\right\|_{L^{p}} \\
& \leq c\left\|\left(\sum_{j \geq 0}\left[2^{s j} \sum_{Q \in \mathcal{V}_{j}} c_{Q}^{\natural} \tilde{\mathbb{1}}_{Q}(\cdot)\right]^{q}\right)^{1 / q}\right\|_{L^{p}} \\
& \leq c\left\|\left(\sum_{j \geq 0}\left[\mathcal{M}_{t}\left(2^{s j} \sum_{Q \in \mathcal{V}_{j}}\left|c_{Q}\right| \tilde{\mathbb{1}}_{Q}\right)(\cdot)\right]^{q}\right)^{1 / q}\right\|_{L^{p}} \\
& \leq c\left\|\left(\sum_{j \geq 0}\left[2^{s j} \sum_{Q \in \mathcal{V}_{j}}\left|c_{Q}\right| \tilde{\mathbb{1}}_{Q}\right]^{q}\right)^{1 / q}\right\|_{L^{p}} \leq c\left\|\left(c_{Q}\right)_{Q}\right\|_{f_{p q}^{s}(\mathcal{Q})} .
\end{aligned}
$$

Thus $\|f\|_{F_{p q}^{s}} \leq c\left\|\left(c_{Q}\right)_{Q}\right\|_{f_{p q}^{s}(\mathcal{Q})}$, which readily implies the unconditional convergence in (3.17) in the norm of $F_{p q}^{s}$.

We next prove an estimate in the opposite direction. Put

$$
\breve{\Psi}_{j}(x):=\sum_{\nu \geq 0} \overline{\psi\left(2 \pi \nu 2^{-j}\right)} e^{2 \pi i \nu x}, \quad j \geq 0, \quad \text { and } \quad \breve{\Psi}_{-1}(x):=1 .
$$

From properties (3.2)-(3.4) of $\psi$ and remark (2) after Theorem 2.3 it follows that

$$
\|f\|_{F_{p q}^{s}} \sim\left\|\left(\sum_{j \geq-1}\left(2^{s j}\left|\breve{\Psi}_{j} * f(\cdot)\right|\right)^{q}\right)^{1 / q}\right\|_{L^{p}} .
$$

Observe that $\breve{\Psi}_{j} * f$ is a trigonometric polynomial of degree $<\frac{4}{3} 2^{j}$ and for $Q \in \mathcal{Q}_{j}$

$$
\begin{aligned}
\left|\left\langle f, G_{Q}\right\rangle\right| & =2^{-j / 2}\left|\breve{\Psi}_{j} * f\left(x_{Q}\right)+\breve{\Psi}_{j} * f\left(x_{Q^{*}}\right)\right| \\
& \leq c 2^{-j / 2}\left(\sup _{y \in Q}\left|\breve{\Psi}_{j} * f(y)\right|+\sup _{y \in Q^{*}}\left|\breve{\Psi}_{j} * f(y)\right|\right) .
\end{aligned}
$$

Using the above and Lemma 3.7 we get

$$
\begin{aligned}
\sum_{Q \in \mathcal{Q}_{j}}\left[\left|\left\langle f, G_{Q}\right\rangle\right| \tilde{\mathbb{1}}_{Q}(\cdot)\right]^{q} & \leq c \sum_{Q \in \mathcal{V}_{j}}\left[\sup _{y \in Q}\left|\breve{\Psi}_{j} * f(y)\right| \mathbb{1}_{Q}(\cdot)\right]^{q} \\
& \leq c\left[\sup _{y \in \mathbb{T}} \frac{\left|\breve{\Psi}_{j} * f(y)\right|}{\left(1+2^{j}|x-y|\right)^{1 / t}}\right]^{q} \leq c\left[\mathcal{M}_{t}\left(\left|\breve{\Psi}_{j} * f\right|\right)(x)\right]^{q} .
\end{aligned}
$$

Further, we use the definition of $\|\cdot\|_{f_{p q}^{s}}$ in (3.14) and the maximal inequality (2.11) to obtain

$$
\begin{aligned}
\left\|\left(\left\langle f, G_{Q}\right\rangle\right)\right\|_{f_{p q}^{s}} & :=\left\|\left(\sum_{j \geq-1} 2^{j s q} \sum_{Q \in \mathcal{Q}_{j}}\left[\left|\left\langle f, G_{Q}\right\rangle\right| \tilde{\mathbb{1}}_{Q}(\cdot)\right]^{q}\right)^{1 / q}\right\|_{L_{p}} \\
& \leq c\left\|\left(\sum_{j \geq-1} 2^{j s q}\left[\mathcal{M}_{t}\left(\left|\breve{\Psi}_{j} * f\right|\right)(\cdot)\right]^{q}\right)^{1 / q}\right\|_{L_{p}} \\
& \leq c\left\|\left(\sum_{j \geq-1}\left[2^{s j}\left|\breve{\Psi}_{j} * f(\cdot)\right|\right]^{q}\right)^{1 / q}\right\|_{L^{p}} \leq c\|f\|_{F_{p q}^{s}} .
\end{aligned}
$$

Here for the last inequality we used (3.21). 


\section{General scheme for Construction of BASes}

4.1. The setting. Assume that $H$ is a separable complex Hilbert space (of functions) and $\mathcal{D} \subset H$ is a linear subspace (of test functions) furnished with a locally convex topology induced by a sequence of norms or semi-norms. Let $\mathcal{D}^{\prime}$ be the dual of $\mathcal{D}$ consisting of all continuous linear functionals on $\mathcal{D}$. We also assume that $H \subset \mathcal{D}^{\prime}$. The pairing of $f \in \mathcal{D}^{\prime}$ and $\phi \in \mathcal{D}$ will be denoted by $\langle f, \phi\rangle:=f(\bar{\phi})$ and we assume that it is consistent with the inner product $\langle f, g\rangle$ in $H$. Typical examples are

(a) $H=L^{2}\left(\mathbb{R}^{n}\right), \mathcal{D}=\mathcal{S}\left(\mathbb{R}^{n}\right)$ the Schwartz class on $\mathbb{R}^{n}$, and $\mathcal{D}^{\prime}=\mathcal{S}^{\prime}$ the dual space of all tempered distributions on $\mathbb{R}^{n}$;

(b) $H=L^{2}\left(\mathbb{R}^{n}\right), \mathcal{D}=\mathcal{S}_{\infty}\left(\mathbb{R}^{n}\right)$ the set of all functions $\phi$ in the Schwartz class $\mathcal{S}\left(\mathbb{R}^{n}\right)$ such that $\int \phi(x) x^{\alpha}=0$ for $\alpha \in \mathbb{Z}_{+}^{n}$, and $\mathcal{D}^{\prime}$ its dual;

(c) $H=H^{2}(\mathbb{T})$ the Hardy space of boundary values of holomorphic functions in the unit disc $D$, and $\mathcal{D}:=D_{+}$and $\mathcal{D}^{\prime}:=\mathcal{D}_{+}^{\prime}$ as described in $\S 2$.

Our next assumption is that $L \subset \mathcal{D}^{\prime}$ with norm $\|\cdot\|_{L}$ is a quasi-Banach space of distributions, which is continuously embedded in $\mathcal{D}^{\prime}$. Further, we assume that $\mathcal{D} \subset H \cap L$ and $\mathcal{D}$ is dense in $H$ and $L$ with respect to their respective norms.

We also assume that $\ell(\mathcal{Q})$ with norm $\|\cdot\|_{\ell(\mathcal{Q})}$ is an associated to $L$ quasi-Banach space of complex-valued sequences with domain a countable index set $\mathcal{Q}$. Coupled with a basis $\Psi$ the sequence space $\ell(\mathcal{Q})$ will be utilized for characterization of the space $L$. In addition to being a quasi-norm we assume that $\|\cdot\|_{\ell(\mathcal{Q})}$ obeys the conditions:

(i) For any sequence $\left(h_{Q}\right)_{Q \in \mathcal{Q}} \in \ell(\mathcal{Q})$ one has $\left\|\left(h_{Q}\right)\right\|_{\ell(\mathcal{Q})}=\left\|\left(\left|h_{Q}\right|\right)\right\|_{\ell(\mathcal{Q})}$.

(ii) If the sequences $\left(h_{Q}\right)_{Q \in \mathcal{Q}},\left(g_{Q}\right)_{Q \in \mathcal{Q}} \in \ell(\mathcal{Q})$ and $\left|h_{Q}\right| \leq\left|g_{Q}\right|$ for $Q \in \mathcal{Q}$, then $\left\|\left(h_{Q}\right)\right\|_{\ell(\mathcal{Q})} \leq c\left\|\left(g_{Q}\right)\right\|_{\ell(\mathcal{Q})}$.

(iii) Compactly supported sequences are dense in $\ell(\mathcal{Q})$.

\subsection{Construction of bases for spaces of distributions.}

4.2.1. The old basis. Given spaces $\mathcal{D} \subset H \subset \mathcal{D}^{\prime}, L$, and $\ell(\mathcal{Q})$ as described in $\S 4.1$ with $\mathcal{Q}$ a countable index set, we assume that $\Psi:=\left\{\psi_{Q}: Q \in \mathcal{Q}\right\} \subset \mathcal{D}$ is an orthonormal basis for $H$, that is, $\left\langle\psi_{Q}, \psi_{P}\right\rangle=\delta_{Q P}$ for $Q, P \in \mathcal{Q}$, and for any $f \in H$

$$
f=\sum_{Q \in \mathcal{Q}}\left\langle f, \psi_{Q}\right\rangle \psi_{Q} \quad \text { in } H \quad \text { and } \quad\|f\|_{H}=\left\|\left(\left\langle f, \psi_{Q}\right\rangle\right)_{Q}\right\|_{\ell^{2}(\mathcal{Q})} .
$$

We also assume that $\Psi$ is a basis for the space $L$ in the following sense: Every $f \in L$ has a unique representation in terms of $\left\{\psi_{Q}\right\}_{Q \in \mathcal{Q}}$ and

$$
f=\sum_{Q \in \mathcal{Q}}\left\langle f, \psi_{Q}\right\rangle \psi_{Q}
$$

where the convergence is unconditional in $L$, and

$$
c_{1}\|f\|_{L} \leq\left\|\left(\left\langle f, \psi_{Q}\right\rangle\right)_{Q}\right\|_{\ell(\mathcal{Q})} \leq c_{2}\|f\|_{L}
$$

for some constants $c_{1}, c_{2}>0$.

Remark 4.1. In (4.1)-(4.2) above and throughout the rest of this section when we write "in $H$ " or "in $L$ " it will always mean that the convergence of the respective series is unconditional in $H$ or in $L$. For unconditional convergence and bases we refer the reader to [10]. 
4.2.2. Construction of a new basis. Our idea is to first construct by perturbing $\Psi$ a new basis $\Theta=\left\{\theta_{Q}: Q \in \mathcal{Q}\right\}$ for $H$ with elements $\theta_{Q} \in H$ and then to show that under some additional localization and approximation conditions $\Theta$ is a basis for $L$. Since $\Psi$ is a basis for $H$, we have

$$
\theta_{Q}=\sum_{P \in \mathcal{Q}}\left\langle\theta_{Q}, \psi_{P}\right\rangle \psi_{P} \quad \text { in } H
$$

Denote by $\mathbf{A}$ the transformation matrix

$$
\mathbf{A}:=\left(a_{Q P}\right)_{Q, P \in \mathcal{Q}}, \quad a_{Q P}:=\left\langle\theta_{Q}, \psi_{P}\right\rangle .
$$

Our key assumption is that the operator $\mathbf{A}$ with matrix $\mathbf{A}$ is bounded and invertible on $\ell^{2}(\mathcal{Q})$ and $\mathbf{A}^{-1}$ is also bounded on $\ell^{2}(\mathcal{Q})$. Observe that if

$$
\mathbf{D}=\left(d_{Q P}\right)_{Q, P \in \mathcal{Q}}:=\left(\left\langle\psi_{Q}-\theta_{Q}, \psi_{P}\right\rangle\right)_{Q, P \in \mathcal{Q}},
$$

then $\mathbf{D}=I-\mathbf{A}$ and, therefore, $\mathbf{A}^{-1}$ exists and is bounded on $\ell^{2}(\mathcal{Q})$ if

$$
\|\mathbf{D}\|_{\ell^{2}(\mathcal{Q}) \mapsto \ell^{2}(\mathcal{Q})}<1 .
$$

This is our main assumption in constructing $\Theta$ as a Riesz basis for $H$. The gist of our method is to approximate $\psi_{Q}$ by $\theta_{Q}$ in such a way that $\mathbf{D}$ satisfies (4.7).

We shall show that under these conditions $\Theta$ is a Riesz basis for $H$. To proceed, let

$$
\mathbf{A}^{-1}=:\left(b_{Q P}\right)_{Q, P \in \mathcal{Q}}
$$

and define

$$
\tilde{\theta}_{Q}:=\sum_{P} \overline{b_{P Q}} \psi_{P}, \quad Q \in \mathcal{Q}
$$

Since $\left(\mathbf{A}^{-1}\right)^{*}=\left(\overline{b_{P Q}}\right)_{Q, P \in \mathcal{Q}}$ is the adjoint matrix of $\mathbf{A}^{-1}$ and

$$
\left\|\left(\mathbf{A}^{-1}\right)^{*}\right\|_{\ell^{2}(\mathcal{Q}) \mapsto \ell^{2}(\mathcal{Q})}=\left\|\mathbf{A}^{-1}\right\|_{\ell^{2}(\mathcal{Q}) \mapsto \ell^{2}(\mathcal{Q})}<\infty
$$

each vector row of $\left(\mathbf{A}^{-1}\right)^{*}$ belongs to $\ell^{2}(\mathcal{Q})$ and hence $\tilde{\theta}_{Q}$ from (4.9) is well defined and $\tilde{\theta}_{Q} \in H$. Evidently, $\overline{b_{P Q}}=\left\langle\tilde{\theta}_{Q}, \psi_{P}\right\rangle$ and hence $\left\langle\psi_{P}, \tilde{\theta}_{Q}\right\rangle=b_{P Q}$.

We set $\tilde{\Theta}:=\left\{\tilde{\theta}_{Q}: Q \in \mathcal{Q}\right\}$. Then it is easy to see that the pair $(\Theta, \tilde{\Theta})$ is a biorthogonal system in $H$, i.e. $\left\langle\theta_{P}, \tilde{\theta}_{Q}\right\rangle=\delta_{Q P}$. Indeed,

$$
\left\langle\theta_{P}, \tilde{\theta}_{Q}\right\rangle=\sum_{I} b_{I Q}\left\langle\theta_{P}, \psi_{I}\right\rangle=\sum_{I} a_{P I} b_{I Q}=\left(\mathbf{A A}^{-1}\right)_{P Q}=\delta_{P Q} .
$$

Proposition 4.2. If $\mathbf{A}, \mathbf{A}^{-1}, \mathbf{A}^{T},\left(\mathbf{A}^{-1}\right)^{T}$ are bounded on $\ell^{2}(\mathcal{Q})$, then $\Theta$ (with dual $\tilde{\Theta})$ is a Riesz basis for $H$, i.e. for any $f \in H$

$$
\begin{gathered}
f=\sum_{Q \in \mathcal{Q}}\left\langle f, \tilde{\theta}_{Q}\right\rangle \theta_{Q} \quad \text { in } H \quad \text { and } \\
c_{1}\|f\|_{H} \leq\left\|\left(\left\langle f, \tilde{\theta}_{Q}\right\rangle\right)_{Q}\right\|_{\ell^{2}(\mathcal{Q})} \leq c_{2}\|f\|_{H} .
\end{gathered}
$$

Proof. It is well know that (see e.g. [21]) a necessary and sufficient condition for $\Theta \subset H$ to be a Riesz basis for $H$ is that $\Theta$ satisfies the conditions:

(i) $\Theta$ is complete in $H$ (the closed span of $\Theta$ is all of $H$ ). 
(ii) There exist constants $c^{\prime}, c^{\prime \prime}>0$ such that for any compactly supported sequence $h=\left(h_{Q}\right)_{Q \in \mathcal{Q}}$ one has

$$
c^{\prime}\|h\|_{\ell^{2}(\mathcal{Q})} \leq\left\|\sum_{Q \in \mathcal{Q}} h_{Q} \theta_{Q}\right\|_{H} \leq c^{\prime \prime}\|h\|_{\ell^{2}(\mathcal{Q})} .
$$

We shall first prove that for any $Q \in \mathcal{Q}$

$$
\psi_{Q}=\sum_{I \in \mathcal{Q}}\left\langle\psi_{Q}, \tilde{\theta}_{I}\right\rangle \theta_{I}=\sum_{I \in \mathcal{Q}} b_{Q I} \theta_{I} \quad \text { in } H .
$$

To this end we shall utilize this lemma:

Lemma 4.3. The operator $T h:=\sum_{Q \in \mathcal{Q}} h_{Q} \theta_{Q}$ is well defined and bounded as an operator from $\ell^{2}(\mathcal{Q})$ into $H$.

Proof. Let $h=\left(h_{Q}\right)_{Q \in \mathcal{Q}}$ be a compactly supported sequence of complex numbers. Then by the boundedness of $\mathbf{A}^{T}$ on $\ell^{2}(\mathcal{Q})$ and (4.1) we have

$$
\begin{aligned}
\|T h\|_{H} & =\left\|\left(\left\langle\sum_{\mathcal{Q} \in \mathcal{Q}} h_{Q} \theta_{Q}, \psi_{P}\right\rangle\right)_{P}\right\|_{\ell^{2}(\mathcal{Q})}=\left\|\left(\sum_{\mathcal{Q} \in \mathcal{Q}} h_{Q}\left\langle\theta_{Q}, \psi_{P}\right\rangle\right)_{P}\right\|_{\ell^{2}(\mathcal{Q})} \\
& \leq c\left\|\mathbf{A}^{T}\right\|_{\ell^{2}(\mathcal{Q}) \mapsto \ell^{2}(\mathcal{Q})}\|h\|_{\ell^{2}(\mathcal{Q})} \leq c\|h\|_{\ell^{2}(\mathcal{Q})} .
\end{aligned}
$$

Since compactly supported sequences are dense in $\ell^{2}(\mathcal{Q})$, it follows that the operator $T$ is bounded as an operator from $\ell^{2}(\mathcal{Q})$ to $H$. It also follows that for any sequence $\left\{h_{Q}\right\} \in \ell^{2}(\mathcal{Q})$ the series $\sum_{Q \in \mathcal{Q}} h_{Q} \theta_{Q}$ converges unconditionally in $\ell^{2}(\mathcal{Q})$.

We now prove (4.13). Since $\mathbf{A}^{-1}=\left(b_{Q P}\right)_{Q, P \in \mathcal{Q}}$ and $\left\|\left(\mathbf{A}^{-1}\right)^{T}\right\|_{\ell^{2}(\mathcal{Q}) \mapsto \ell^{2}(\mathcal{Q})}<\infty$, we have $\left(b_{Q I}\right)_{I} \in \ell^{2}(\mathcal{Q})$ and applying Lemma 4.3 it follows that $g_{Q}:=\sum_{I \in \mathcal{Q}} b_{Q I} \theta_{I}$ is a well defined element of $H$. On the other hand,

$$
\left\langle g_{Q}, \psi_{P}\right\rangle=\sum_{I \in \mathcal{Q}} b_{Q I}\left\langle\theta_{I}, \psi_{P}\right\rangle=\sum_{I \in \mathcal{Q}} b_{Q I} a_{I P}=\left(\mathbf{A}^{-1} \mathbf{A}\right)_{Q P}=\delta_{Q P}
$$

yielding $g_{Q}=\psi_{Q}$. Hence, (4.13) holds.

As a basis $\Psi:=\left\{\psi_{Q}\right\}$ is complete in $H$ and now (4.13) implies that $\Theta:=\left\{\theta_{Q}\right\}$ is complete in $H$ as well.

We now turn to the proof of (4.12). Let $h=\left(h_{Q}\right)_{Q \in \mathcal{Q}}$ be a compactly supported sequence of complex numbers. Then by Lemma $4.3\left\|\sum_{Q \in \mathcal{Q}} h_{Q} \theta_{Q}\right\|_{H} \leq c\|h\|_{\ell^{2}(\mathcal{Q})}$, which gives the right-hand side estimate in (4.12).

For the other direction, denote briefly $f:=\sum_{Q \in \mathcal{Q}} h_{Q} \theta_{Q}$. As was shown above the system $\tilde{\Theta}:=\left\{\tilde{\theta}_{Q}\right\}$, defined in (4.9), is the dual of $\Theta$ and hence for $Q \in \mathcal{Q}$

$$
h_{Q}=\left\langle f, \tilde{\theta}_{Q}\right\rangle=\left\langle f, \sum_{P \in \mathcal{Q}} \overline{b_{P Q}} \psi_{P}\right\rangle=\sum_{P \in \mathcal{Q}} b_{P Q}\left\langle f, \psi_{P}\right\rangle,
$$

which yields

$$
\|h\|_{\ell^{2}(\mathcal{Q})} \leq\left\|\left(\mathbf{A}^{-1}\right)^{T}\right\|_{\ell^{2}(\mathcal{Q}) \mapsto \ell^{2}(\mathcal{Q})}\left\|\left(\left\langle f, \psi_{P}\right\rangle\right)_{P}\right\|_{\ell^{2}(\mathcal{Q})} \leq c\|f\|_{H}
$$

Here we used the boundedness of $\left(\mathbf{A}^{-1}\right)^{T}$ on $\ell^{2}(\mathcal{Q})$ and (4.1). Thus (4.12) is established and hence $\Theta$ is a Riesz basis. This in turn implies (4.10)-(4.11).

Our next aim is to show that under some reasonable conditions on $\mathbf{A}$ and $\mathbf{A}^{-1}$ the system $\Theta$ is an unconditional basis for $L$. 
Theorem 4.4. Let $\mathbf{A}$ and $\mathbf{A}^{-1}$ be bounded on $\ell^{2}(\mathcal{Q})$ and assume that the operators $\mathbf{A}^{T}$ and $\left(\mathbf{A}^{-1}\right)^{T}$ with matrices $\mathbf{A}^{T}$ and $\left(\mathbf{A}^{-1}\right)^{T}$ are bounded on $\ell(\mathcal{Q})$. Then $\Theta$ (with dual $\tilde{\Theta}$ ) is a basis for $L$ in the following sense: Each $f \in L$ has a unique representation in terms of $\left\{\theta_{Q}\right\}_{Q \in \mathcal{Q}}$ and

$$
f=\sum_{Q \in \mathcal{Q}}\left\langle f, \tilde{\theta}_{Q}\right\rangle \theta_{Q}
$$

where by definition $\left\langle f, \tilde{\theta}_{Q}\right\rangle:=\sum_{P \in \mathcal{Q}}\left\langle f, \psi_{P}\right\rangle\left\langle\psi_{P}, \tilde{\theta}_{Q}\right\rangle$ and the series converges unconditionally in L. Furthermore, there exist constants $c_{1}, c_{2}>0$ such that

$$
c_{1}\|f\|_{L} \leq\left\|\left(\left\langle f, \tilde{\theta}_{Q}\right\rangle\right)_{Q}\right\|_{\ell(\mathcal{Q})} \leq c_{2}\|f\|_{L} \quad \text { for } \quad f \in L .
$$

Proof. We first prove the right-hand side estimate in (4.15). Let $f \in L$. Then by (4.9) $\left\langle\psi_{Q}, \tilde{\theta}_{P}\right\rangle=b_{Q P}$ and using that $\left(\mathbf{A}^{-1}\right)^{T}$ is bounded on $\ell(\mathcal{Q})$, we get

$$
\begin{aligned}
\left\|\left(\left\langle f, \tilde{\theta}_{Q}\right\rangle\right)\right\|_{\ell(\mathcal{Q})} & =\left\|\left(\sum_{P \in \mathcal{Q}}\left\langle f, \psi_{P}\right\rangle\left\langle\psi_{P}, \tilde{\theta}_{Q}\right\rangle\right)_{Q}\right\|_{\ell(\mathcal{Q})} \leq\left\|\left(\sum_{P \in \mathcal{Q}} b_{P Q}\left\langle f, \psi_{P}\right\rangle\right)_{Q}\right\|_{\ell(\mathcal{Q})} \\
& \leq\left\|\left(\mathbf{A}^{-1}\right)^{T}\right\|_{\ell(\mathcal{Q}) \mapsto \ell(\mathcal{Q})}\left\|\left(\left\langle f, \psi_{Q}\right\rangle\right)_{Q}\right\|_{\ell(\mathcal{Q})} \leq c\|f\|_{L},
\end{aligned}
$$

where for the last inequality we used (4.3). Thus the claimed inequality is confirmed.

We next deal with the left-hand side estimate in (4.15). For this we first prove that the operator $T h:=\sum_{Q \in \mathcal{Q}} h_{Q} \theta_{Q}$ is well defined and bounded as an operator from $\ell(\mathcal{Q})$ into $L$. Let first $h=\left(h_{Q}\right)_{Q \in \mathcal{Q}}$ be a compactly supported sequence of complex numbers. By (4.4) we have $\theta_{Q}=\sum_{P} a_{Q P} \psi_{P}$ and hence

$$
T h=\sum_{Q \in \mathcal{Q}} h_{Q} \theta_{Q}=\sum_{P \in \mathcal{Q}}\left(\sum_{Q \in \mathcal{Q}} a_{Q P} h_{Q}\right) \psi_{P} .
$$

Then by (4.3) and the boundedness of $\mathbf{A}^{T}$ on $\ell(\mathcal{Q})$, we obtain

$$
\|T h\|_{L} \leq c\left\|\left(\sum_{\mathcal{Q} \in \mathcal{Q}} a_{Q P} h_{Q}\right)_{P}\right\|_{\ell(\mathcal{Q})} \leq c\left\|\mathbf{A}^{T}\right\|_{\ell(\mathcal{Q}) \mapsto \ell(\mathcal{Q})}\|h\|_{\ell(\mathcal{Q})} \leq c\|h\|_{\ell(\mathcal{Q})} .
$$

By condition (iii) on $\ell(\mathcal{Q})$ compactly supported sequences are dense in $\ell(\mathcal{Q})$, and hence the operator $T$ can be extended uniquely as a bounded operator from $\ell(\mathcal{Q})$ to $L$. More precisely, from above and conditions (i)-(iii) on $\ell(\mathcal{Q})$ it follows that for any sequence $h=\left(h_{Q}\right)_{Q \in \mathcal{Q}} \in \ell(\mathcal{Q})$ and any $\varepsilon>0$ there exists a finite set of indices $\mathcal{F} \subset \mathcal{Q}$ such that for every index set $\mathcal{F}^{\prime} \subset \mathcal{Q} \backslash \mathcal{F}$ we have

$$
\left\|\sum_{Q \in \mathcal{F}^{\prime}} h_{Q} \theta_{Q}\right\|_{L}<\varepsilon
$$

This readily implies (see [10]) that the series $\sum_{Q \in \mathcal{Q}} h_{Q} \theta_{Q}$ converges unconditionally in $L$. Thus $T$ is a well defined and bounded operator from $\ell(\mathcal{Q})$ into $L$. Assuming that (4.14) is valid, using (4.17) we obtain the left-hand side estimate in (4.15).

It remains to show the validity of (4.14). We define a new operator $U$ on $L$ by

$$
U f:=\sum_{Q \in \mathcal{Q}}\left\langle f, \tilde{\theta}_{Q}\right\rangle \theta_{Q}
$$

By (4.16) we have $\left(\left\langle f, \tilde{\theta}_{Q}\right\rangle\right)_{Q \in \mathcal{Q}} \in \ell(\mathcal{Q})$ for $f \in L$. Then from the boundedness of the operator $T$ it follows that the operator $U$ is well defined and bounded on $L$. On the other hand by (4.10), $U f=f$ for $f \in H$ and hence for $f \in \mathcal{D}$, but $\mathcal{D}$ is dense in $L$. Therefore, $U f=f$ for $f \in L$, i.e. (4.14) holds true. 


\section{Rational basis for B- AND F-Spaces on the DisC}

In this section we utilize the scheme from $\S 4$ to the construction of a basis consisting of rational functions of uniformly bounded degrees for Besov and TriebelLizorkin spaces of holomorphic functions in the unit disc. Our basis will be of the form $\left\{R_{Q}\right\}_{Q \in \mathcal{Q}}$, where the index set $\mathcal{Q}$ is the family of dyadic cubes defined in (3.7).

\subsection{Construction of the new basis. Let}

$$
\Phi(x):=\frac{1}{\left(1+x^{2}\right)^{n}}, \quad x \in \mathbb{R}, n \in \mathbb{N},
$$

and denote

$$
\Theta_{K}:=\left\{\theta: \theta(x)=\sum_{\nu=1}^{K} c_{\nu} \Phi\left(a x+b_{\nu}\right), c_{\nu}, b_{\nu} \in \mathbb{R}, a>0\right\} .
$$

Evidently, $\Theta_{K} \subset \mathrm{R}_{2 n K}(\mathbb{R})$, where $\mathrm{R}_{n}(\mathbb{R})$ is the set of all rational functions of degree (order) $\leq n$ on $\mathbb{R}$ with real coefficients.

For future references, we denote by $\mathrm{R}_{n}(D)$ the set of all rational functions $R$ of degree (order) $\leq n$ which are holomorphic on $D$, i.e. the poles of $R$ are outside $D$.

The gist of our method is to construct a rational basis for spaces of holomorphic functions in $D$ by taking the $H^{2}$ projections of the periodized appropriate rational functions from $\mathrm{R}_{K}(\mathbb{R})$ for some fixed $K$.

The first step of this scheme is to approximate Meyer's mother wavelet $\psi$ and its derivatives by a rational function on $\mathbb{R}$ :

Proposition 5.1. Suppose $\psi$ is the function from (3.2) - (3.4) which generates Meyer's orthonormal basis of $L^{2}(\mathbb{R})$. Given $N, n \in \mathbb{N}, M>0$ with $2 n>M$, and $\varepsilon>0$ there exists $K \geq 1$ and $\theta \in \Theta_{K}$ such that

$$
\text { (ii) } \quad \int_{\mathbb{R}} x^{r} \theta(x) d x=0, \quad 0 \leq r \leq N \text {. }
$$

For the construction of a function $\theta$ as above we refer the reader to [7, Theorem 4.1] (see also [8, Theorem 3.8]).

We are now prepared to define the elements of our rational basis. Just as in the construction of the two hump wavelet basis of $H^{2}(\mathbb{T})$ (see (3.5)) we define for each $Q=Q_{j k} \in \mathcal{Q}_{j}\left(0 \leq k<2^{j-1}, j \geq 0\right)$ a function $R_{Q}$ on $\mathbb{T}$ by

$$
R_{Q}(x):=2^{-j / 2} \sum_{\nu \geq 0} \hat{\theta}\left(2 \pi \nu 2^{-j}\right)\left(e^{2 \pi i \nu\left(x-k 2^{-j}\right)}+e^{2 \pi i \nu\left(x-k^{*} 2^{-j}\right)}\right),
$$

where $k^{*}=2^{j}-k-1$ and we set $R_{Q}(x):=1$ if $Q=Q_{-1,0}$. Here $\hat{\theta}$ is the Fourier transform of $\theta$. Then

$$
\mathcal{R}:=\left\{R_{Q}: Q \in \mathcal{Q}\right\}
$$

is our new system on $\mathbb{T}$. The parameters $N, M, n, \varepsilon$ of $\mathcal{R}$ will be selected in Theorem 5.2 below. 
5.2. Main result. In the following, we let $\mathcal{J}:=1 / \min \{1, p\}$ in the case of B-spaces and $\mathcal{J}:=1 / \min \{1, p, q\}$ for F-spaces. Now, we can state our main result:

Theorem 5.2. Suppose $s \in \mathbb{R}, 0<p, q<\infty$, and let $\mathcal{R}:=\left\{R_{Q}\right\}_{Q \in \mathcal{Q}}$ be constructed as above with $\theta$ satisfying (5.3), where $N>\max \{s, \mathcal{J}, \mathcal{J}-s-1\}$ and $M>N+1$. Then

(a) Each $R_{Q} \in \mathcal{R}$ extends to a rational function in $\mathrm{R}_{K}(D)$ for some fixed $K<\infty$.

(b) If $\varepsilon>0$ is sufficiently small (depending only on $N$ and $M$ ) the system $\mathcal{R}$ has a dual system $\tilde{\mathcal{R}}$ such that $(\mathcal{R}, \tilde{\mathcal{R}})$ is a Riesz basis for $H^{2}(\mathbb{T})$ and unconditional basis for $B_{p q}^{s}$ and $F_{p q}^{s}$ in the sense of Theorem 4.4. In particular, any $f \in B_{p q}^{s}$ or $f \in F_{p q}^{s}$ has a unique representation

$$
f=\sum_{Q \in \mathcal{Q}} d_{Q}(f) R_{Q}
$$

where $d_{Q}(f):=\left\langle f, \tilde{R}_{Q}\right\rangle:=\sum_{P \in \mathcal{Q}}\left\langle f, G_{P}\right\rangle\left\langle G_{P}, \tilde{R}_{Q}\right\rangle$ and the convergence is unconditional in $B_{p q}^{s}$ or $F_{p q}^{s}$. Furthermore, if $f \in B_{p q}^{s}$ or $f \in F_{p q}^{s}$ we have

$$
\|f\|_{B_{p q}^{s}} \sim\left\|\left(d_{Q}(f)\right)_{Q}\right\|_{b_{p q}^{s}(\mathcal{Q})} \quad \text { or } \quad\|f\|_{F_{p q}^{s}} \sim\left\|\left(d_{Q}(f)\right)_{Q}\right\|_{f_{p q}^{s}(\mathcal{Q})},
$$

respectively.

Remark 5.3. (a) The roles of $\{s, p, q\}$ and $\{N, M, \varepsilon\}$ above can be reversed, i.e. the conditions on the parameters of the basis $\mathcal{R}$ can be considered as conditions on the indices $\{s, p, q\}$ under which the conclusion of the theorem holds.

(b) Using the identification from (2.3) - (2.4) one can immediately extend the elements of $\mathcal{R}$ to $D$ to obtain a rational basis for the $F$ - and B-spaces of holomorphic functions on $D$. In the future, e.g. in $\S 6$, we shall denote this basis by $\mathcal{R}$ again.

We shall carry out the proof of Theorem 5.2 in several steps, showing first that the elements of $\mathcal{R}$ are rational functions.

5.3. The basis elements are rational functions. In this subsection we prove part (a) of Theorem 5.2 (see Proposition 5.6 below). To this end we need some preparation.

Denote by $\mathrm{P}_{n}$ the set of all univariate algebraic polynomials of degree $\leq n$.

Lemma 5.4. Let $\Phi(x)=\frac{1}{\left(1+x^{2}\right)^{n}}, n \in \mathbb{N}$. Then there exists a polynomial $P \in \mathrm{P}_{n-1}$ such that

$$
\hat{\Phi}(x)=e^{-|x|} P(|x|),
$$

where $\hat{\Phi}$ is the Fourier transform of $\Phi$ (see (3.1)).

Proof. Since $\Phi$ is an even function $\hat{\Phi}$ is also even, and hence it suffices to establish (5.8) for $x>0$ only. Changing the variables we get

$$
\hat{\Phi}(x)=\int_{\mathbb{R}} \frac{1}{\left(1+y^{2}\right)^{n}} e^{-i x y} d y=x^{2 n-1} \int_{\mathbb{R}} \frac{e^{i y}}{\left(x^{2}+y^{2}\right)^{n}} d y .
$$

Let

$$
f(z):=\frac{e^{i z}}{\left(x^{2}+z^{2}\right)^{n}}=\frac{e^{i z}}{(z-i x)^{n}(z+i x)^{n}} .
$$


Fix $x>0$ and for $R>x$ let $\gamma=\gamma_{R}$ be the curve consisting of the segment $[-R, R]$ on the real line and the arch of the circle $|z|=r$ in the upper half plane. By the Residue Theorem

$$
\frac{1}{2 \pi i} \int_{\gamma} f(z) d z=\operatorname{Res}(f ; i x)
$$

where $\operatorname{Res}(f ; i x)$ is the residue of $f$ at $i x$ given by

$$
\begin{aligned}
\operatorname{Res}(f ; i x) & =\frac{1}{(n-1) !} \lim _{z \rightarrow i x} \frac{d^{n-1}}{d z^{n-1}}(z-i x)^{n} f(z) \\
& =\frac{1}{(n-1) !} \lim _{z \rightarrow i x} \frac{d^{n-1}}{d z^{n-1}}\left(\frac{e^{i z}}{(z+i x)^{n}}\right) .
\end{aligned}
$$

Using Leibniz's formula there exist constants $a_{\nu} \in \mathbb{C}, \nu=0, \ldots, n-1$, such that

$$
\operatorname{Res}(f ; i x)=e^{-x} \sum_{\nu=0}^{n-1} a_{\nu} x^{-n-\nu}
$$

Writing

$$
\int_{\gamma} f(z) d z=\int_{-R}^{R} \frac{e^{i y}}{\left(x^{2}+y^{2}\right)^{n}} d y+\int_{0}^{\pi} \frac{e^{i R e^{i t}} i R e^{i t}}{\left(x^{2}+R^{2} e^{2 i t}\right)^{n}} d t
$$

we observe that the second integral above tends to zero as $R \rightarrow \infty$ and letting $R \rightarrow \infty$ we arrive at

$$
\int_{\mathbb{R}} \frac{e^{i y}}{\left(x^{2}+y^{2}\right)^{n}} d y=2 \pi i \operatorname{Res}(f ; i x) .
$$

Finally, putting together (5.9), (5.10), and (5.11) we get

$$
\hat{\Phi}(x)=e^{-x} \sum_{\nu=0}^{n-1} c_{\nu} x^{\nu}
$$

for some constants $c_{0}, \ldots, c_{n-1} \in \mathbb{C}$, which completes the proof.

Lemma 5.5. Let $P \in \mathrm{P}_{n-1}, n \in \mathbb{N}$, and $a \in \mathbb{C}$. Then there exist constants $b_{0}, \ldots, b_{n-1} \in \mathbb{C}$ such that

$$
\sum_{m \geq 0} P(m)\left(\frac{z}{a}\right)^{m}=\sum_{\nu=0}^{n-1} b_{\nu}\left(\frac{a}{a-z}\right)^{\nu+1}, \quad|z|<|a| .
$$

Proof. Put $p_{\nu}(x):=\frac{(x+1) \cdots(x+\nu)}{\nu !}$ for $\nu \geq 1$ and $p_{0}(x):=1$. Evidently, the polynomials $p_{0}, \ldots, p_{n-1}$ are linear independent and $p_{\nu}(m)=\left(\begin{array}{c}m+\nu \\ \nu\end{array}\right)$. Therefore, there exist coefficients $b_{\nu} \in \mathbb{C}, \nu=0, \ldots, n-1$, such that

$$
P(m)=\sum_{\nu=0}^{n-1} b_{\nu}\left(\begin{array}{c}
m+\nu \\
\nu
\end{array}\right), \quad m \in \mathbb{Z} .
$$

On the other hand, the identity $\left(\frac{1}{a-z}\right)^{(\nu)}=\frac{\nu !}{(a-z)^{\nu+1}}$ implies that for $|z|<|a|$

$$
\frac{a^{\nu+1}}{(a-z)^{\nu+1}}=\frac{a^{\nu}}{\nu !} \sum_{n \geq 0}\left(\frac{z^{n}}{a^{n}}\right)^{(\nu)}=\sum_{n \geq \nu}\left(\begin{array}{c}
n \\
\nu
\end{array}\right)\left(\frac{z}{a}\right)^{n-\nu}=\sum_{m \geq 0}\left(\begin{array}{c}
m+\nu \\
\nu
\end{array}\right)\left(\frac{z}{a}\right)^{m} .
$$


Using this and (5.13) we obtain

$$
\sum_{m \geq 0} P(m)\left(\frac{z}{a}\right)^{m}=\sum_{\nu=0}^{n-1} b_{\nu} \sum_{m \geq 0}\left(\begin{array}{c}
m+\nu \\
\nu
\end{array}\right)\left(\frac{z}{a}\right)^{m}=\sum_{\nu=0}^{n-1} b_{\nu}\left(\frac{a}{a-z}\right)^{\nu+1},
$$

which confirms (5.12).

We are now prepared to show that the new system $\mathcal{R}=\left\{R_{Q}: Q \in \mathcal{Q}\right\}$ (defined in (5.5)) consists of rational functions of uniformly bounded degrees.

Proposition 5.6. Each element $R_{Q}$ of the new system $\mathcal{R}$ extends to a rational function from $\mathrm{R}_{2 n K}(D)$.

Proof. Let $Q=Q_{j k} \in \mathcal{Q}_{j}\left(0 \leq k<2^{j-1}, j \geq 0\right)$. From the definition of $R_{Q}$ in (5.4) we infer that $R_{Q}$ can be extended in $D$ as

$$
R_{Q}(z)=H_{j, k}(z)+H_{j, k^{*}}(z),
$$

where

$$
H_{j, k}(z)=\sum_{m \geq 0} \hat{\theta}\left(2 \pi m 2^{-j}\right) e^{-2 \pi i m k 2^{-j}} z^{m}, \quad|z|<1,
$$

and $k^{*}:=2^{j}-k-1$. Observe that from the decay of $\hat{\theta}$ it follows that the last series above converges absolutely for $|z|=1$. Thus it suffices to show that $H_{j, k}(z) \in \mathrm{R}_{n K}$ if $0 \leq k<2^{j}$.

Since $\theta \in \Theta_{K}$ then $\theta$ is of the form $\theta(x)=\sum_{\nu=1}^{K} c_{\nu} \Phi\left(\alpha x+\beta_{\nu}\right)$ with $\alpha>0$ and $c_{\nu}, \beta_{\nu} \in \mathbb{R}$. Applying the Fourier transform and Lemma 5.4 we get

$$
\hat{\theta}(\xi)=\alpha^{-1} \hat{\Phi}(\xi / \alpha) \sum_{\nu=1}^{K} c_{\nu} e^{i\left(\beta_{\nu} / \alpha\right) \xi}=\alpha^{-1} e^{-|\xi| / \alpha} P(|\xi| / \alpha) \sum_{\nu=1}^{K} c_{\nu} e^{i\left(\beta_{\nu} / \alpha\right) \xi}
$$

where $P \in \mathrm{P}_{n-1}$, and hence

$$
\begin{aligned}
H_{j, k}(z) & =\sum_{m \geq 0} \alpha^{-1} e^{-2 \pi m 2^{-j} \alpha^{-1}} P\left(2 \pi m 2^{-j} \alpha^{-1}\right) \sum_{\nu=1}^{K} c_{\nu} e^{i 2 \pi m 2^{-j}\left(\beta_{\nu} / \alpha-k\right)} z^{m} \\
& =\sum_{\nu=1}^{K} \frac{c_{\nu}}{\alpha} \sum_{m \geq 0} P\left(2 \pi m 2^{-j} \alpha^{-1}\right)\left(\frac{z}{\exp \left\{2 \pi 2^{-j} \alpha^{-1}-i 2 \pi 2^{-j}\left(\frac{\beta_{\nu}}{\alpha}-k\right)\right\}}\right)^{m} .
\end{aligned}
$$

We set $a_{\nu}:=\exp \left\{2 \pi 2^{-j} \alpha^{-1}-i 2 \pi 2^{-j}\left(\beta_{\nu} / \alpha-k\right)\right\}$ and apply Lemma 5.5 to obtain

$$
H_{j, k}(z)=\sum_{\nu=1}^{K} \alpha^{-1} c_{\nu} \sum_{m \geq 0} P\left(2 \pi m 2^{-j} \alpha^{-1}\right)\left(\frac{z}{a_{\nu}}\right)^{m}=\sum_{\nu=1}^{K} \sum_{r=0}^{n-1} \gamma_{r} c_{\nu}\left(\frac{a_{\nu}}{a_{\nu}-z}\right)^{r+1},
$$

for some $\gamma_{0}, \ldots, \gamma_{n-1} \in \mathbb{C}$. Observe that $\left|a_{\nu}\right|>1$. Therefore, $H_{j, k} \in \mathrm{R}_{n K}(D)$, which completes the proof.

5.4. Almost diagonal operators. For the proof of Theorem 5.2 we need to study the boundedness of almost diagonal operators on the sequence spaces $b_{p q}^{s}:=b_{p q}^{s}(\mathcal{Q})$ and $f_{p q}^{s}:=f_{p q}^{s}(\mathcal{Q})$.

Definition 5.7. Let $\mathbf{A}$ be a linear operator acting on $b_{p q}^{s}$ or $b_{p q}^{s}$ with associated matrix $\left(a_{Q P}\right)_{Q, P \in \mathcal{Q}}$. We say that $\mathbf{A}$ is almost diagonal if there exists $\delta>0$ such that

$$
\sup _{Q, P \in \mathcal{Q}} \frac{\left|a_{Q P}\right|}{\omega_{\delta}(Q, P)}<\infty
$$


where for $Q \in \mathcal{Q}_{j}$ and $P \in \mathcal{Q}_{i}, i, j \geq-1$,

$\omega_{\delta}(Q, P):=2^{(i-j) s} \min \left\{2^{(i-j)(1+\delta) / 2}, 2^{(j-i)(\mathcal{J}+\delta / 2-1 / 2)}\right\}$

$$
\times\left(\frac{1}{\left(1+2^{\min \{i, j\}}\left|x_{Q}-x_{P}\right|\right)^{\mathcal{J}+\delta}}+\frac{1}{\left(1+2^{\min \{i, j\}}\left|x_{Q}-x_{P^{*}}\right|\right)^{\mathcal{J}+\delta}}\right)
$$

with $\mathcal{J}:=1 / \min \{1, p\}$ for $b_{p q}^{s}$ and $\mathcal{J}:=1 / \min \{1, p, q\}$ for $f_{p q}^{s}$.

Note that above we use our earlier convention $|x-y|:=\min _{n \in \mathbb{Z}}|x-y+n|$.

We claim that almost diagonal operators are bounded on $b_{p q}^{s}$ and $f_{p q}^{s}$. More precisely, with the notation

$$
\|\mathbf{A}\|_{\delta}:=\sup _{Q, P \in \mathcal{Q}} \frac{\left|a_{Q P}\right|}{\omega_{\delta}(Q, P)}
$$

the following result holds:

Proposition 5.8. Suppose $s \in \mathbb{R}, 0<q \leq \infty$, and $0<p<\infty(0<p \leq \infty$ in the case of b-spaces) and assume that $\|\mathbf{A}\|_{\delta}<\infty$ for some $\delta>0$. Then there exists a constant $c>0$ such that for any sequence $h:=\left\{h_{Q}\right\}_{Q \in \mathcal{Q}} \in b_{p q}^{s}$

$$
\|\mathbf{A} h\|_{b_{p q}^{s}} \leq c\|\mathbf{A}\|_{\delta}\|h\|_{b_{p q}^{s}}
$$

and for any $h:=\left\{h_{Q}\right\}_{Q \in \mathcal{Q}} \in f_{p q}^{s}$

$$
\|\mathbf{A} h\|_{f_{p q}^{s}} \leq c\|\mathbf{A}\|_{\delta}\|h\|_{f_{p q}^{s}} .
$$

The proof of this proposition is quite similar to the proof of Theorem 3.3 in [4]. We omit it.

5.5. Estimation of inner products. Our next step is to estimate the decay of $\left|\left\langle R_{Q}, G_{P}\right\rangle\right|$ and $\left|\left\langle G_{Q}-R_{Q}, G_{P}\right\rangle\right|$ away from the main diagonal. To this end we need some preparation.

Lemma 5.9. Let $h \in C^{2}(\mathbb{R})$ satisfy the following conditions for some $N>1$ and $M>N+1$ :

$$
\begin{aligned}
& \int_{\mathbb{R}} x^{r} h(x) d x=0, \quad 0 \leq r \leq N, \\
& \left|h^{(r)}(x)\right| \leq A(1+|x|)^{-M}, \quad r=0,1,2 .
\end{aligned}
$$

If

$$
g(x)=\sum_{\nu \geq 0} \hat{h}\left(2 \pi \nu n^{-1}\right) e^{2 \pi i \nu x}, \quad n \geq 1,
$$

then

$$
|g(x)| \leq c A n(1+n|x|)^{-N}, \quad|x| \leq 1 / 2
$$

Proof. Formally,

$$
\xi^{m} \hat{h}^{(r)}(\xi)=(-i)^{m} i^{r} \int_{\mathbb{R}}\left(x^{r} h(x)\right)^{(m)} e^{-i \xi x} d x
$$

and hence, using (5.21) and the assumption $M>N+1$, we get

$$
|\xi|^{2}\left|\hat{h}^{(r)}(\xi)\right| \leq \int_{\mathbb{R}}\left|\left(x^{r} h(x)\right)^{\prime \prime}\right| d x \leq c A \quad \text { for } \quad 0 \leq r \leq N,
$$


which implies

$$
\left|\hat{h}^{(r)}(\xi)\right| \leq c A(1+|\xi|)^{-2}, \quad \xi \in \mathbb{R}, 0 \leq r \leq N .
$$

On the other hand, by (5.20) we have $\hat{h}^{(r)}(0)=0$ for $0 \leq r \leq N$.

Let $f:=\left(\hat{h}(\cdot) \mathbb{1}_{[0, \infty)}\right)^{\vee}$, i.e. $f(x)=\int_{0}^{\infty} \hat{h}(\xi) e^{i \xi x} d \xi$. Using the above we infer

$$
|x|^{N}|f(x)|=\left|\int_{0}^{\infty} \hat{h}^{(N)}(\xi) e^{i \xi x} d \xi\right| \leq \int_{0}^{\infty}\left|\hat{h}^{(N)}(\xi)\right| d \xi \leq c A
$$

and hence

$$
|f(x)| \leq c A(1+|x|)^{-N}, \quad x \in \mathbb{R}
$$

The Poisson's summation formula

$$
\sum_{\nu \in \mathbb{Z}} f(x+\nu)=\sum_{\nu \in \mathbb{Z}} \hat{f}(2 \pi \nu) e^{2 \pi i \nu x}
$$

applied to $f(n \cdot)$ (observe that $\widehat{f(n \cdot)}=n^{-1} \hat{f}(\cdot / n)$ ), yields

$$
g(x)=\sum_{\nu \geq 0} \hat{h}\left(2 \pi \nu n^{-1}\right) e^{2 \pi i \nu x}=n \sum_{\nu \in \mathbb{Z}} f(n(x+\nu)) .
$$

This coupled with (5.23) gives, for $|x| \leq 1 / 2$,

$|g(x)| \leq c n A \sum_{\nu \in \mathbb{Z}}(1+n|x+\nu|)^{-N} \leq c n A(1+n|x|)^{-N} \sum_{\nu \geq 1} \nu^{-N} \leq c n A(1+n|x|)^{-N}$,

which completes the proof.

In the following, for a given function $g$ on $\mathbb{R}$ we set $g_{j}(x):=2^{j} g\left(2^{j} x\right)$ for $j \in \mathbb{Z}$. Observe that $\left(g_{j}\right)^{\wedge}(\xi)=\hat{g}\left(\xi 2^{-j}\right)$.

Lemma 5.10. Suppose the functions $g \in C^{N}(\mathbb{R})$ and $h \in C(\mathbb{R})$ satisfy the conditions:

$$
\left|g^{(r)}(x)\right| \leq A_{1}(1+|x|)^{-M_{1}}, \quad 0 \leq r \leq N, \quad|h(x)| \leq A_{2}(1+|x|)^{-M_{2}},
$$

and

$$
\int_{\mathbb{R}} x^{r} h(x) d t=0 \quad \text { for } 0 \leq r \leq N-1,
$$

where $N \geq 1, M_{2} \geq M_{1}, M_{2}>N+1$, and $A_{1}, A_{2}>0$. Then for $k \geq j(j, k \in \mathbb{Z})$

$$
\left|g_{j} * h_{k}(x)\right| \leq c A_{1} A_{2} 2^{-(k-j) N_{2}} 2^{j}\left(1+2^{j}|x|\right)^{-M_{1}},
$$

where $c>0$ depends only on $M_{1}, M_{2}$, and $N$.

The proof of this lemma is essentially the same as the proof of Lemma B.1 in [4]. The only difference is in the normalization of the functions. We omit the proof.

To give our main assertion here we introduce the following notation: Given $f, h \in L^{1}(\mathbb{R})$ we write for $Q=Q_{j, k} \in \mathcal{Q}_{j}$ and $P=P_{m, l} \in \mathcal{Q}_{m}$

$$
\begin{aligned}
& F_{Q}(x):=2^{-j / 2} \sum_{\nu \geq 0} \hat{f}\left(2 \pi \nu 2^{-j}\right)\left(e^{2 \pi i \nu\left(x-k 2^{-j}\right)}+e^{2 \pi i \nu\left(x-k^{*} 2^{-j}\right)}\right), \\
& H_{P}(x):=2^{-m / 2} \sum_{\nu \geq 0} \hat{h}\left(2 \pi \nu 2^{-m}\right)\left(e^{2 \pi i \nu\left(x-l 2^{-m}\right)}+e^{2 \pi i \nu\left(x-l^{*} 2^{-m}\right)}\right) .
\end{aligned}
$$


Proposition 5.11. Suppose $g, h \in C^{N+2}(\mathbb{R}), N \geq 2$, satisfy the conditions:

$$
\left|f^{(r)}(x)\right| \leq A_{1}(1+|x|)^{-M} \text { and }\left|h^{(r)}(x)\right| \leq A_{2}(1+|x|)^{-M}
$$

for $0 \leq r \leq N+2$, where $M>N+1$, and

$$
\int_{\mathbb{R}} x^{r} f(x) d x=\int_{\mathbb{R}} x^{r} h(x) d x=0, \quad 0 \leq r \leq N-1 .
$$

Then for $Q=Q_{j, k} \in \mathcal{Q}_{j}$ and $P=P_{m, l} \in \mathcal{Q}_{m}$

$$
\left|\left\langle F_{Q}, H_{P}\right\rangle\right| \leq c A_{1} A_{2} 2^{-|j-m|(N+1 / 2)}
$$

$$
\times\left[\left(1+2^{\min \{j, m\}}\left|x_{Q}-x_{P}\right|\right)^{-N}+\left(1+2^{\min \{j, m\}}\left|x_{Q}-x_{P^{*}}\right|\right)^{-N}\right],
$$

where $c>0$ depends only on $N$ and $M$.

Proof. From the definition of $F_{Q}, H_{P}$ in (5.25) we get

$$
\begin{aligned}
\left\langle F_{Q}, H_{P}\right\rangle & =2^{-(j+m) / 2} \sum_{\nu=0}^{\infty} \hat{f}\left(\frac{2 \pi \nu}{2^{j}}\right) \overline{\hat{h}\left(\frac{2 \pi \nu}{2^{m}}\right)} e^{2 \pi i \nu\left(x_{P}-x_{Q}\right)} \\
& +2^{-(j+m) / 2} \sum_{\nu=0}^{\infty} \hat{f}\left(\frac{2 \pi \nu}{2^{j}}\right) \overline{\hat{h}\left(\frac{2 \pi \nu}{2^{m}}\right)} e^{2 \pi i \nu\left(x_{\left.P^{*}-x_{Q}\right)}\right.} \\
& +2^{-(j+m) / 2} \sum_{\nu=0}^{\infty} \hat{f}\left(\frac{2 \pi \nu}{2^{j}}\right) \overline{\hat{h}\left(\frac{2 \pi \nu}{2^{m}}\right)} e^{2 \pi i \nu\left(x_{P}-x_{Q^{*}}\right)} \\
& +2^{-(j+m) / 2} \sum_{\nu=0}^{\infty} \hat{f}\left(\frac{2 \pi \nu}{2^{j}}\right) \overline{\hat{h}\left(\frac{2 \pi \nu}{2^{m}}\right)} e^{2 \pi i \nu\left(x_{\left.P^{*}-x_{Q^{*}}\right)}\right.} \\
& =: \beta_{1}+\beta_{2}+\beta_{3}+\beta_{4} .
\end{aligned}
$$

Assume that $m \geq j$. It is readily seen that

$$
\hat{f}\left(\frac{2 \pi \nu}{2^{j}}\right) \overline{\hat{h}\left(\frac{2 \pi \nu}{2^{m}}\right)}=\left(f_{j} * \tilde{h}_{m}\right)^{\wedge}(2 \pi \nu)=\left(f * \tilde{h}_{m-j}\right)^{\wedge}\left(\frac{2 \pi \nu}{2^{j}}\right),
$$

where $\tilde{h}(x):=\overline{h(-x)}$. On the other hand, evidently

$$
\left(f * \tilde{h}_{m-j}\right)^{(r)}(x)=\left(f^{(r)} * \tilde{h}_{m-j}\right)(x) .
$$

By (5.26) we have

$$
\left|f^{(r+s)}(x)\right| \leq A_{1}(1+|x|)^{-M}, 0 \leq s \leq N, 0 \leq r \leq 2 \text {, and }|\tilde{h}(x)| \leq A_{2}(1+|x|)^{-M} .
$$

Also, by (5.27) it follows that $\int_{\mathbb{R}} x^{s} \tilde{h}(x) d x=0,0 \leq s \leq N-1$. We apply Lemma 5.10 to $f^{(r)}$ and $\tilde{h}$ to obtain

$$
\left|\left(f * \tilde{h}_{m-j}\right)^{(r)}(x)\right|=\left|\left(f^{(r)} * \tilde{h}_{m-j}\right)(x)\right| \leq c 2^{-(m-j) N}(1+|x|)^{-M}, \quad 0 \leq r \leq 2 .
$$

On the other hand by (5.27) it follows that

$$
\int_{\mathbb{R}} x^{s}\left(f * \tilde{h}_{m-j}\right)^{(r)}(x) d x=0, \quad 0 \leq r \leq 2, \quad 0 \leq s \leq 2(N-1), \quad(2(N-1) \geq N) .
$$

Using the above we apply Proposition 5.9 to $f * \tilde{h}_{m-j}$ and infer

$$
\begin{aligned}
\beta_{1} & \leq c 2^{-(j+m) / 2} 2^{-(m-j) N} 2^{j}\left(1+2^{j}\left|x_{P}-x_{Q}\right|\right)^{-N} \\
& =c 2^{-(m-j)(N+1 / 2)}\left(1+2^{j}\left|x_{Q}-x_{P}\right|\right)^{-N} .
\end{aligned}
$$


Exactly in the same way we get similar estimates for $\beta_{2}, \beta_{3}, \beta_{4}$, where $x_{Q}-x_{P}$ is replaced by $x_{Q}-x_{P^{*}}, x_{Q^{*}}-x_{P}$, and $x_{Q^{*}}-x_{P^{*}}$, respectively. These estimates imply (5.28) taking into account that $2^{j}\left|x_{P}-x_{Q}\right| \sim 2^{j}\left|x_{P^{*}}-x_{Q^{*}}\right|$ and $2^{j}\left|x_{P^{*}}-x_{Q}\right| \sim$ $2^{j}\left|x_{P}-x_{Q^{*}}\right|$.

\subsection{Proof of Theorem 5.2. Let}

$$
\mathbf{A}:=\left(a_{Q P}\right)_{Q, P \in \mathcal{Q}}, \quad a_{Q, P}:=\left\langle R_{Q}, G_{P}\right\rangle,
$$

and $\mathbf{D}=I-\mathbf{A}$, that is,

$$
\mathbf{D}:=\left(d_{Q P}\right)_{Q, P \in \mathcal{Q}}, \quad d_{Q P}:=\left\langle G_{Q}-R_{Q}, G_{P}\right\rangle .
$$

Evidently, Theorem 5.2 will follow by Theorem 4.4, applied with $H:=H^{2}(\mathbb{T})$, $L:=B_{p q}^{s}$ and $\ell(\mathcal{Q}):=b_{p q}^{s}(\mathcal{Q})\left(\right.$ or $L:=F_{p q}^{s}$ and $\left.\ell(\mathcal{Q}):=f_{p q}^{s}(\mathcal{Q})\right)$ and $\Psi:=\mathcal{G}$, Meyer's biorthogonal basis from (3.8) and Theorem 3.3, if we prove that the matrices $\mathbf{A}, \mathbf{A}^{-1}$ above are bounded on $H^{2}(\mathbb{T})$ and $\mathbf{A}^{T},\left(\mathbf{A}^{-1}\right)^{T}$ are bounded on $b_{p q}^{s}\left(\right.$ or $\left.f_{p q}^{s}\right)$.

To establish the boundedness of $\mathbf{A}, \mathbf{A}^{T}$ it is sufficient to show that $\mathbf{A}$ is almost diagonal on $b_{p q}^{s}\left(f_{p q}^{s}\right)$, while for the boundedness of $\mathbf{A}^{T},\left(\mathbf{A}^{-1}\right)^{T}$ it is sufficient to prove that $\|\mathbf{D}\|_{b_{p q}^{s} \rightarrow b_{p q}^{s}}<1$ (or $\|\mathbf{D}\|_{f_{p q}^{s} \rightarrow f_{p q}^{s}}<1$ ). However, by Proposition 5.8 $\|\mathbf{D}\|_{b_{p q}^{s} \rightarrow b_{p q}^{s}} \leq c\|\mathbf{D}\|_{\delta}$, and hence it suffices to prove that there exists $\delta>0$ such that for any sufficiently small $\varepsilon>0$

$$
\|\mathbf{D}\|_{\delta}<\varepsilon
$$

and similarly for $f_{p q}^{s}$. We shall only prove (5.31); the proof of the estimates $\|\mathbf{A}\|_{\delta},\left\|\mathbf{A}^{T}\right\|_{\delta}<\infty$ is very similar and will be omitted.

By the definition of the systems $\left\{G_{Q}\right\}$ and $\left\{R_{Q}\right\}$ in (3.8) and (5.5), we have for $Q=Q_{j k} \in \mathcal{Q}_{j}(j \geq 0)$

$$
\begin{aligned}
G_{Q}(x)-R_{Q}(x) & =2^{-j / 2} \sum_{\nu \geq 0}(\psi-\theta)^{\wedge}\left(2 \nu \pi 2^{-j}\right)\left(e^{2 \nu \pi i\left(x-k 2^{-j}\right)}+e^{2 \nu \pi i\left(x-k^{*} 2^{-j}\right)}\right), \\
G_{Q}(x) & =2^{-j / 2} \sum_{\nu \geq 0} \hat{\psi}\left(2 \nu \pi 2^{-j}\right)\left(e^{2 \nu \pi i\left(x-k 2^{-j}\right)}+e^{2 \nu \pi i\left(x-k^{*} 2^{-j}\right.}\right),
\end{aligned}
$$

and from the construction of $\theta$ in Proposition 5.1 and from (3.2)

$$
\begin{gathered}
\left|(\psi-\theta)^{(r)}(x)\right| \leq \varepsilon(1+|x|)^{-M}, \quad\left|\psi^{(r)}(x)\right| \leq c(1+|x|)^{-M}, \quad 0 \leq r \leq N+2, \\
\int_{\mathbb{R}} x^{r} \theta(x) d x=0, \int_{\mathbb{R}} x^{r} \psi(x) d x=0, \quad 0 \leq r \leq N-1,
\end{gathered}
$$

where $M>N+1$ and $N>\max \{s, \mathcal{J}, \mathcal{J}-s-1\}$. Now, applying Proposition 5.11 we obtain for $Q \in \mathcal{Q}_{j}, P \in \mathcal{Q}_{i}, i, j \geq 0$,

$$
\begin{aligned}
\left|\left\langle G_{Q}-R_{Q}, G_{P}\right\rangle\right| & \leq c \varepsilon \min \left\{2^{(j-i)}, 2^{(i-j)}\right\}^{N+\frac{1}{2}} \\
& \left.\times\left((1+32) \quad 2^{\min \{i, j\}}\left|x_{Q}-x_{P}\right|\right)^{-N}+\left(1+2^{\min \{i, j\}}\left|x_{Q}-x_{P^{*}}\right|\right)^{-N}\right) .
\end{aligned}
$$

It is easy to see that this estimate also holds whenever $i=-1$ or $j=-1$. Therefore, there exists $\delta>0$ such that $\|\mathbf{D}\|_{\delta}<c \varepsilon$. However, $\varepsilon$ is independent of $c, N, M$, and hence $c \varepsilon$ can be replaced by $\varepsilon$, i.e. (5.31) holds. 
5.7. Localization and norms of the basis elements $\left\{\boldsymbol{R}_{Q}\right\}$. The following result will be instrumental in the next section.

Proposition 5.12. For any $Q \in \mathcal{Q}$ we have

$$
\left|R_{Q}(x)\right| \leq c \ell(Q)^{-1 / 2}\left(\left(1+2^{j}\left|x-x_{Q}\right|\right)^{-N}+\left(1+2^{j}\left|x-x_{Q^{*}}\right|\right)^{-N}\right)
$$

and if $\varepsilon>0$ in the definition of $R_{Q}$ is sufficiently small

$$
\left\|R_{Q}\right\|_{L^{p}} \sim \ell(Q)^{-1 / 2+1 / p} \text { for } 1 / N<p \leq \infty .
$$

Proof. Estimate (5.33) is immediate from the definition of $G_{Q}$ in (5.4) and Lemma 5.9.

From (5.33) it readily follows that $\left\|R_{Q}\right\|_{L^{p}} \leq c \ell(Q)^{-1 / 2+1 / p}$ if $p>1 / N$. The estimate in the other direction is more subtle. Exactly as in the proof of (5.32) using Proposition 5.11 we get $\left\|G_{Q}-R_{Q}\right\|_{L^{2}} \leq c \varepsilon$. On the other hand $\left\|G_{Q}\right\|_{L^{2}}=1$. Therefore, choosing $\varepsilon>0$ so that $c \varepsilon \leq 1 / 2$ we obtain $1 / 2 \leq\left\|R_{Q}\right\|_{L^{2}} \leq 3 / 2$. This and (5.33) imply (5.34) in general. Indeed, if $1 / N<p<2$, then

$$
1 / 4 \leq\left\|R_{Q}\right\|_{L^{2}}^{2} \leq\left\|R_{Q}\right\|_{L^{p}}^{p}\left\|R_{Q}\right\|_{L^{\infty}}^{2-p} \leq c\left\|R_{Q}\right\|_{L^{p}}^{p} \ell(Q)^{-1+p / 2},
$$

and hence $\left\|R_{Q}\right\|_{L^{p}} \geq c \ell(Q)^{-1 / 2+1 / p}$. If $2<p<\infty$ we apply Hölder's inequality to obtain

$$
1 / 4 \leq \int_{\mathbb{T}}\left|R_{Q}(x)\right|^{2} d x \leq\left\|R_{Q}\right\|_{L^{p}}\left\|R_{Q}\right\|_{L^{p^{\prime}}} \leq c\left\|R_{Q}\right\|_{L^{p}} \ell(Q)^{-1 / 2+1 / p^{\prime}},
$$

which leads again to $\left\|R_{Q}\right\|_{L^{p}} \geq c \ell(Q)^{-1 / 2+1 / p}$. For $p=\infty$ this estimate is immediate from $\left\|R_{Q}\right\|_{L^{2}} \geq 1 / 2$.

\section{Rational approximation in the Hardy spaces $H^{p}$ on the disC}

Here we use the development of a rational basis from the previous section to give another proof of the direct estimate of A. Pekarskii [16] for rational approximation in $H^{p}(D)$. Given $f \in H^{p}$ we denote by $\rho_{n}\left(f, H^{p}\right)$ the best approximation of $f$ from the class $\mathrm{R}_{n}(D)$ of rational functions of degree $\leq n$ on $D$, i.e.

$$
\rho_{n}\left(f, H^{p}\right):=\inf _{g \in \mathrm{R}_{n}(D)}\|f-g\|_{H^{p}} .
$$

Pekarskii's result involves the Besov space $B_{\tau}^{s}:=B_{\tau \tau}^{s}, s>0,1 / \tau:=s+1 / p$, $0<p<\infty$, of holomorphic functions in $D$.

Theorem 6.1 (Pekarskii [16] ). For any $f \in B_{\tau}^{s}$

$$
\rho_{n}\left(f, H^{p}\right) \leq c n^{-s}\|f\|_{B_{\tau}^{s}}, \quad n \geq 1,
$$

where the constant $c>0$ depends only on $s$ and $p$.

This result coupled with the companion Bernstein estimate from [15] enabled Pekarskii to give a complete characterization of the rates of rational approximation (approximation spaces) in the $H^{p}$ spaces, see [14] for a comprehensive discussion of these results.

To prove estimate (6.1) we shall consider $n$-term approximation in $H^{p}$ from the rational basis $\mathcal{R}:=\left\{R_{Q}: Q \in \mathcal{Q}\right\}$ we constructed in $\S 5.1$. More precisely, we shall use $\left\{R_{Q}\right\}$ to denote both the basis functions introduced in (5.4)-(5.5) and their analytic extensions to $D$ that are rational functions with poles outside $D$ as shown in $\S 5.3$. As such we have $\left\|R_{Q}\right\|_{H^{p}(D)}=\left\|R_{Q}\right\|_{L^{p}}$, where on the right we have 
the $L^{p}$-norm of $R_{Q}$ on $\mathbb{T}$. We hope this slight abuse of notation will not create problems.

Let $\Sigma_{n}$ be the nonlinear set of all functions $g$ of the form

$$
g=\sum_{Q \in \Lambda_{n}} a_{Q} R_{Q}
$$

where $\Lambda_{n} \subset \mathcal{Q}, \# \Lambda_{n} \leq n$, and $\Lambda_{n}$ may vary with $g$. Denote by $\sigma_{n}(f)_{p}$ the error of best $H^{p}$-approximation to $f \in H^{p}$ from $\Sigma_{n}$, i.e.

$$
\sigma_{n}\left(f, H^{p}\right):=\inf _{g \in \Sigma_{n}}\|f-g\|_{H^{p}} .
$$

The approximation will take place in $H^{p}(D), 1 / N<p<\infty$, where $N$ is the main parameter of the basis $\mathcal{R}$, see Theorem $5.2 ; N$ can be selected arbitrarily large.

Under this assumption, from (5.7) in Theorem 5.2 and (5.34) one obtains easily the following representation of the norm in $B_{\tau}^{s}$ :

$$
\|f\|_{B_{\tau}^{s}} \sim\left(\sum_{Q \in \mathcal{Q}}\left\|\left\langle f, \tilde{R}_{Q}\right\rangle R_{Q}\right\|_{H^{p}}^{\tau}\right)^{1 / \tau} .
$$

We now give the main result of this section.

Theorem 6.2. If $f \in B_{\tau}^{s}$, then

$$
\sigma_{n}\left(f, H^{p}\right) \leq c n^{-s}\|f\|_{B_{\tau}^{s}}, \quad n \geq 1,
$$

where $c$ depends only on $s, p$, and the parameters of $\mathcal{R}$.

Remark 6.3. Observe that by Theorem 5.2 $R_{Q} \in \mathrm{R}_{K}(D)$ for all $Q \in \mathcal{Q}$ with some $K=$ const. Therefore, $\Sigma_{n} \subset \mathrm{R}_{K n}(D)$ and hence $\rho_{n}\left(f, H^{p}\right) \leq \sigma_{K n}\left(f, H^{p}\right)$. Consequently, estimate (6.3) implies Pekarskii's estimate (6.1).

The proof of Theorem 6.2 relies on the following lemma.

Lemma 6.4. Let $F=\sum_{Q \in \Omega_{n}} a_{Q} R_{Q}$, where $\Omega_{n} \subset \mathcal{Q}$ and $\# \Omega_{n} \leq n$. Suppose $\left\|a_{Q} R_{Q}\right\|_{H^{p}} \leq A$ for $Q \in \Omega_{n}$, where $0<p<\infty$. Then $\|F\|_{H^{p}} \leq c A n^{1 / p}$.

Proof. Let $1<p<\infty$; the case when $0<p \leq 1$ is trivial. For the rest of the proof we shall work as before with the norms on $\mathbb{T}$ rather than on $D$. By (5.34) and the assumption $\left\|a_{Q} R_{Q}\right\|_{L^{p}} \leq A$ we get $\left|a_{Q}\right| \leq c A \ell(Q)^{1 / 2-1 / p}$ for $Q \in \mathcal{Q}_{j}$. Choose $t$ so that $1 / N<t<1$. Then using (2.12) and (5.33) we infer

$$
\left|R_{Q}(x)\right| \leq c \mathcal{M}_{t}\left(\ell(Q)^{-1 / 2} \mathbb{1}_{Q}\right)(x)+c \mathcal{M}_{t}\left(\ell\left(Q^{*}\right)^{-1 / 2} \mathbb{1}_{Q *}\right)(x), \quad Q \in \mathcal{Q}_{j},
$$

and hence, using the maximal inequality (2.11),

$$
\begin{aligned}
\|F\|_{L^{p}} & \leq c\left\|\sum_{Q \in \Omega_{n}} \mathcal{M}_{t}\left(\left|a_{Q}\right| \ell(Q)^{-1 / 2} \mathbb{1}_{Q}\right)\right\|_{L^{p}}+c\left\|\sum_{Q \in \Omega_{n}} \mathcal{M}_{t}\left(\left|a_{Q}\right| \ell\left(Q^{*}\right)^{-1 / 2} \mathbb{1}_{Q}^{*}\right)\right\|_{L^{p}} \\
& \leq c\left\|\sum_{Q \in \Omega_{n}}\left|a_{Q}\right| \ell(Q)^{-1 / 2} \mathbb{1}_{Q}\right\|_{L^{p}}+c\left\|\sum_{Q \in \Omega_{n}}\left|a_{Q}\right| \ell\left(Q^{*}\right)^{-1 / 2} \mathbb{1}_{Q}^{*}\right\|_{L^{p}} \\
& \leq c A\left\|\sum_{Q \in \Omega_{n}} \ell(Q)^{-1 / p} \mathbb{1}_{Q}\right\|_{L^{p}} .
\end{aligned}
$$

Let $E:=\cup_{Q \in \Omega_{n}} Q$ and $\omega(x):=\min \left\{\ell(Q): Q \in \Omega_{n}, x \in Q\right\}$ for $x \in E$. Evidently, for $x \in Q, Q \in \Omega_{n}$, we have

$$
\sum_{P: x \in P, Q \subset P}(\ell(P) / \ell(Q))^{1 / p} \leq \sum_{\nu \geq 0} 2^{-\nu / p}=c_{1}<\infty .
$$


Therefore,

$$
\sum_{Q \in \Omega_{n}} \ell(Q)^{-1 / p} \mathbb{1}_{Q}(x) \leq c_{1} \omega(x)^{-1 / p}, \quad x \in E
$$

which implies

$$
\begin{aligned}
\|F\|_{L^{p}} & \leq c A\left\|\omega(\cdot)^{-1 / p}\right\|_{L^{p}}=c A\left(\int_{E} \omega(x)^{-1} d x\right)^{1 / p} \\
& \leq c A\left(\sum_{Q \in \Omega_{n}} \ell(Q)^{-1} \int_{E} \mathbb{1}_{Q}(x) d x\right)^{1 / p} \leq c A\left(\# \Omega_{n}\right)^{1 / p} \leq c A n^{1 / p} .
\end{aligned}
$$

One carries out the proof Theorem 6.2 precisely as the proof of Theorem 6.2 in [9], using (6.2) and Lemma 6.4. We omit the details.

\section{REFERENCES}

[1] S. Bochkarev, Existance of a basis in the spaces of analytic functions in the disk and some properties of Franklin's system, Mat. Sbornik, 95(135) (1974), 1-16.

[2] T. M. Flett, Lipschitz spaces of functions on the circle and the disc, J. Math. Anal. Appl. 39 (1972), 125-158.

[3] M. Frazier, B. Jawerth, Decomposition of Besov Spaces, Indiana Univ. Math. J. 34 (1985), 777-799.

[4] M. Frazier, B. Jawerth, A discrete transform and decompositions of distribution, J. of Funct. Anal. 93 (1990), 34-170.

[5] M. Frazier, B. Jawerth, and G. Weiss, Littlewood-Paley Theory and the Study of Function Spaces, CBMS 79 (1991), AMS.

[6] G. Kyriazis, P. Petrushev, On the construction of frames for Treibel-Lizorkin and Besov spaces, Proc. Amer. Math. Soc. 134 (2006), 1759-1770.

[7] G. Kyriazis, P. Petrushev, New Bases for Treibel-Lizorkin and Besov spaces, Trans. Amer. Math. Soc. 354 (2002), 749-776.

[8] G. Kyriazis, P. Petrushev, On the construction of frames for spaces of distributions, J. Funct. Anal. 257 (2009), 2159-2187.

[9] F. J. Narcowich, P. Petrushev, and J. D. Ward, Decomposition of Besov and TriebelLizorkin spaces on the sphere, J. Funct. Anal. 238(2006), 530-564.

[10] J. Lindenstrauss, L. Tsafriri, Classical Banach spaces I, Springer Verlag, 1977.

[11] Y. Meyer, Ondelletes et Opérateurs I: Ondelletes, Hermann, Paris, 1990.

[12] P. Oswald, On Besov-Hardy-Sobolev spaces of analytic functions in the unit disc, Chech. Math. Jour. 33 (108) (1983), 408-426. [http://dml.cz/dmlcz/101891]

[13] J. Peetre, New thoughts on Besov spaces, Duke Univ. Math. Series, Durham, N.C., 1993.

[14] J. Peetre, J. Karlsson, Rational approximation - analysis of work of Pekarskii, Rocky Mountain J. Math. 19 (1989), 313-334.

[15] A. Pekarskii, Inequalities of Bernstein type for derivatives of rational functions and inverese theorems of rational approximation, Mat. Sb. 124 (1984), 571-588.

[16] A. Pekarskii, Classes of analytic functions defined by best rational approximation in $H_{p}$, Mat. Sb. 127 (1985), 3-20.

[17] P. Petrushev, Bases consisting of rational functions of uniformly bounded degrees or more general functions, J. Funct. Anal. 174 (2000), 18-75.

[18] E. Stein, Harmonic analysis: real-variable methods, orthogonality, and oscillatory integrals, Princeton University Press, Princeton, NJ, 1993.

[19] H. Triebel, Theory of function spaces, Monographs in Math. Vol. 78, Birkhäuser, Verlag, Basel, 1983.

[20] H. Triebel, Theory of function spaces II, Monographs in Math., Vol. 84, Birkhäuser, Verlag, Basel, 1992.

[21] R. Young, An introduction to nonharmonic Fourier series, Academic Press, New York, 1980.

[22] A. Zygmund, Trigonometric series, Vol. 1, 2, Cambridge Univ. Press, 1959. 
Department of Mathematics and Statistics, University of Cyprus, 1678 Nicosia, Cyprus

E-mail address: kyriazis@ucy.ac.cy

Department of Mathematics, University of South Carolina, Columbia, SC 29208, and Institute of Mathematics and Informatics, Bulgarian Academy of Sciences

E-mail address: pencho@math.sc.edu 